\title{
Radiotherapy Improves the Prognosis in Signet Ring Cell Carcinoma and Non-Signet Ring Cell Gastric Adenocarcinoma Patients: A SEER Database Analysis
}

\author{
Wang C ${ }^{1 \#}$, Li H ${ }^{2 \#}$, Luo J ${ }^{3 \#}$, Liu $\mathrm{R}^{4}$, Ma $\mathrm{T}^{5}$ and $\mathrm{Yu} \mathrm{L}^{1 *}$ \\ 1Departments of Intervention, The People's Hospital of \\ Guangxi Zhuang Autonomous Region, Nanning, Guangxi, \\ China \\ ${ }^{2}$ Center for Digestive Diseases, The Seventh Affiliated \\ Hospital of Sun Yat-Sen University, Shenzhen, \\ Guangdong, China \\ ${ }^{3}$ Guangxi Huiren Medical Technology Co., Ltd, Nanning, \\ Guangxi, China \\ ${ }^{4}$ Departments of Hepatobiliary and Endocrine Surgery, \\ The People's Hospital of Guangxi Zhuang Autonomous \\ Region, Nanning, Guangxi, China \\ ${ }^{5}$ School of Pharmaceutical Sciences \& Key Laboratory \\ of Advanced Pharmaceutical Technology, Ministry of \\ Education, Zhengzhou University, Zhengzhou, China \\ "Contributed equally to this Work \\ *Corresponding author: Lei Yu, Departments of \\ Intervention, The People's Hospital of Guangxi Zhuang \\ Autonomous Region, Nanning, Guangxi 530021, China
}

Received: J une 17, 2021; Accepted: July 12, 2021; Published: July 19, 2021

\begin{abstract}
Objective: To investigate the effect of radiotherapy on prognosis in gastric signet ring cell carcinoma (SRCC) and gastric non-signet ring cell adenocarcinoma (NSRCC) patients.
\end{abstract}

Methods: A total of 23,971 patients diagnosed with gastric cancer were collected from 2004 to 2013 by SEER database, including 17,679 NSRCC patients and 6,292 SRCC patients. Patients were divided to two groups and compared according to whether radical gastrectomy was performed on them. After clinic pathological characteristics of the two groups were balanced by the propensity score matching (PSM) method, survival rates of the two groups were then compared.

Results: The Over Survival (OS) and Cancer-Specific Survival (CSS) time of SRCC and NSRCC patients in the radiotherapy groupwere significantly better than those in the non-radiotherapy group. But the clinical and pathological characteristics (gender, age, pathological differentiation degree, $T$ stage, $\mathrm{N}$ stage, and metastasis) of the two groups were significantly unbalanced $(p<0.001)$. After 1:1 PSM matching, it was found that radiotherapy had no significant effect on OS and CSS time of NSRCC patients, but radiotherapy could significantly improve OS and CSS time of SRCC patients.

Conclusion: SRCC patients are more sensitive to radiotherapy than NSRCC patients. Although the prognosis of SRCC is far less than that of NSRCC patients, the treatment strategy of surgical resection of primary lesions combined with radiotherapy should be recommend for SRCC patients, and whether radiotherapy benefits NSRCC patients still needs to be further studied.

Keywords: Over Survival; NSRCC; Cancer-Specific Survival; gastric cancer; SEER; Radiotherapy

\section{Abbreviations}

SRCC: Signet Ring Cell Carcinoma; NSRCC: Non-Signet Ring Cell Adenocarcinoma; PSM: Propensity Score Matching; OS: Over Survival; CSS: Cancer-Specific Survival Time

\section{Introduction}

Gastric Cancer (GC) is the fourth - common malignant tumor in the world and is the third leading cause of cancer-related death [1]. Gastric carcinoma has many histological types and classifications, such as Lauren [2], Japanese [3] and Ming classifications [4]. Among of all these classifications, each histological type has its own characteristics. Therefore, different histological types of gastric cancer might determine they have different treatment strategies. Adenocarcinoma is the most common pathological type of gastric cancer and it includes a special type named as Signet Ring Cell Carcinoma gastric carcinoma (SRCC), which accounts for 15.1-28.2 \% of gastric cancer [5]. SRCC was once thought to be a histological type characterized with poor prognosis and high possibility of infiltrating the stomach [6]. Therefore, SRCC has been widely reported as an independent predictor of poor prognosis especially in Western countries. The majority of these tumors are diagnosed at an advanced stage, with higher lymph node metastasis rates, higher incidence of peritoneal cancer and lower chemotherapy sensitivity [7]. SRCC is considered a unique pathological entity in the pathological types of gastric cancer. Some studies have shown that SRCC is always associated with the rejuvenation, higher stage of differentiation and lower survival rate compared to gastric adenocarcinoma [8].

In recent years, treatment levels in gastric cancer patients have been rapidly improved especially for local advanced gastric cancers. The United States NCCN Guide or the European ESMO Guidelines recommended perioperative radiotherapy and chemotherapy treatment model, so the therapeutic effect of local advanced gastric cancer has been improved obviously. With the development and widespread popularization of D2 surgery, the indications of radiotherapy and the scope of radiotherapy have become the scholars' focus [9]. Radiotherapy can be used for early shrinkage of tumors before surgery and can helpimprove the likelihood of therapeutic resection [10]. In gastric cancer patients with distant metastasis, it is recommended that some treatment methods such as irradiating the primary focus or metastatic cooker to reduce the obstruction, 
compression, bleeding or pain should be carried out in order to improve the life quality of patients. A large amount of samples data show that adjuvant radiotherapy can effectively improve the survival and prognosis of gastric adenocarcinoma patients [11]. In clinical practices, it could be found that the effects of radiotherapy in NonSignet Rings Cell Gastric Adenocarcinoma (NSRCC) and SRCC patients are different. A retrospective study on two large samples found that in phase II and III, rectal ring cell carcinoma patients undergoing radiotherapy had a statistically worse CSS time than rectal non-ring cell carcinoma patients [12], indicating that in SRCC and NSRCC patients there might also be differences in the sensitivity to radiotherapy. In order to explore the effect of radiotherapy on gastric SRCC and NSRCC patients, we selected and studied the patients with gastric cancer through SEER database and divided them into SRCC and NSRCC patients according to the pathological types of gastric cancer. By comparing the effect of radiotherapy on the survival and prognosis of SRCC and NSRCC patients, we can provide a basis for clinical rational application of radiotherapy.

\section{Materials and Methods}

\section{Patient samples collection}

In this study, we investigated the clinical value of radiotherapy for SRCC and NSRCC patients using SEER database (http://seer. cancer.gov/about/overview.html), which is maintained by the National Cancer Institute and includes 18 population-based cancer registries with information of cancer morbidity and mortality in the United States [13]. This version of the SEER database we used was released in April 2014 (submitted in November 2013). All TNM classifications are defined according to the criteria described in the Cancer staging manual of the United States Joint Commission on Cancer (AJCC) (6th edition 2004, Paras. I, II, III and IV). Histological classifications were divided into well differentiation (G1), moderately differentiation (G2), poor differentiation (G3) and un-differentiation (G4) groups. CSS time was calculated from the diagnosis beginning date to the specific death date of tumor patients or the end date of follow-up. SEER database includes the diagnostic information such as age, sex, race, years of diagnosis, numbers of tumors, tumors size, TNM staging, histological type, histological classification, surgical and radiotherapy status for each patient. Tumors are classified according to the International Classification of Tumor Diseases (ICD-O). We obtained 150,265 patients who were encoded as the primary site of "stomach", excluding patients without pathological diagnosis $(n=7432)$, patients without pathological staging $(n=40193)$, patients with pathology as simple parasites $(n=167)$, patients with pathological diagnosis of cancer only $(n=2656)$, patients with pathological diagnosis of neuroendocrine tumor $(n=1604)$, patients lack of TMN staging $(n=74242)$. Finally, 23,971 patients was included in our study. Of these, there are 17,679 NSRCC patients and 6,292 SRCC patients. The groupcomparison was carried out according to whether radical resection was performed on these patients.

\section{Ethics Statement}

This study is conducted based on data from the public SEER database, which does not contain any identifiers. Due to the retrospective nature of the study, the patient's informed consent is not required. The analysis does not involve interactions with human subjects or use their personally identifiable information. Prior to the analysis, patient records/information are anonymous and deidentified, and these methods are in accordance with the guidelines of the Ethics Committee of the People's Hospital of Guangxi Zhuang Autonomous Region. This study protocol was approved by the Ethics Committee of the People's Hospital of Guangxi Zhuang Autonomous Region.

\section{Statistical analysis}

Compare classification variables between two treatment groups using Pearson's $\chi^{2}$ for statistical analysis, while discontinuous variables use ANOVA. The survival curve is calculated by KaplanMeier method and the Log-rank test is compared. When the survival curve is intersected and $p<0.05$, the intersection is obtained by landmark. Discovery and the segmented statistical analysis is carried out according to the pvalue of each segment [14]. Cox proportional risk regression model is used to identify the risk factors that affects CSS and OS time independently in gastric SRCC and NSRCC patients. The effect of radiotherapy on the survival prognosis of SRCC and NSRCC is compared by tendency score matching analysis (propensity score-matched analyses, PSM). The nearest neighbor matching method is used to complete the one-to-one match (age, sex, race, years of diagnosis, numbers of tumors, tumors size, TNM staging, histological type, histological classification and other variables) without replacement. Caliper width is 0.05 times the standard deviation of the tendentious score logit, and it is estimated that the co-construction variable deviation of $>99 \%$ can be eliminated [15].

\section{Results}

\section{Patient characteristics}

A total of 23971 GC patients were included in this study, most of them were diagnosed with NSRCC $(n=17679,73.75 \%)$. There are more people diagnosed at age $<65$ years in the SRCC groupthan in the NSRCC group $(54.52 \%$ vs. $37.89 \%, \mathrm{p}<0.001)$. The occurrence frequency of low differentiation tumors in SRCC patients was also higher than that in NSRCC patients $(97.35 \%$ vs. $59.66 \%, \mathrm{p}<0.001)$. And the frequency of lymph node metastasis in SRCC patients was higher than that in NSRCC patients $(57.54 \%$ vs. $52.39 \%, \mathrm{p}<0.001)$. Moreover, the frequency of distant metastasis in SRCC patients was higher than that in NSRCC patients $(45.01 \%$ vs. $26.39 \%$, p $<0.001)$. In addition, the SRCC groupcontained more female patients $(48.90 \% v s$. $31.01 \%, \mathrm{p}<0.001$ ) (Table 1-4).

\section{Prognostic factors in SRCC and NSRCC patients}

The multivariate analysis results of all SRCC and NSRCC patients using Cox model are shown in Table 5 and 6. From the data, we can see that older $(>65$ years old), larger the tumor size $(>1 \mathrm{~cm})$ and lower the degree of tumor differentiation lead to shorter survival time of SRCC and NSRCC patients ( $\mathrm{p}<0.001)$. Besides, whether to carry out gastrectomy or radiotherapy and distant metastasis degree are also important factors affecting the survival time in SRCC and NSRCC patients. However, each factor has some different effects on SRCC and NSRCC patients. For example, the degree of tumor differentiation is not an independent risk factor affecting the prognosis of SRCC patients, but it is an independent risk factor for NSRCC patients. For both SRCC and NSRCC patients, radiotherapy has prolonged their survival time obviously, but there are still some differences between them. The univariate analysis of OS time show that radiotherapy for 
Table 1: Demographics according to radiotherapy vs. non-radiotherapy for NSRCC patients without gastrectomy.

\begin{tabular}{|c|c|c|c|c|c|c|c|c|c|c|}
\hline & \multicolumn{5}{|c|}{ Unadjusted } & \multicolumn{5}{|c|}{ Adjusted } \\
\hline & Non-rad & SD $/ \%$ & Rad & SD $/ \%$ & $p$ & Non-rad & SD/\% & Rad & SD $/ \%$ & $p$ \\
\hline No. & 5157 & $68.82 \%$ & 2336 & $31.18 \%$ & - & 709 & $50 \%$ & 709 & $50 \%$ & - \\
\hline \multicolumn{11}{|l|}{ Gender } \\
\hline Male & 3397 & $65.87 \%$ & 1811 & $77.53 \%$ & \multirow{2}{*}{$<0.001$} & 598 & $84.34 \%$ & 598 & $84.34 \%$ & \multirow{2}{*}{0.990} \\
\hline Female & 1760 & $34.13 \%$ & 525 & $22.47 \%$ & & 111 & $15.66 \%$ & 111 & $15.66 \%$ & \\
\hline \multicolumn{11}{|l|}{ Age (yrs) } \\
\hline$\geq 65$ & 1828 & $35.45 \%$ & 823 & $35.23 \%$ & \multirow{2}{*}{0.856} & 217 & $30.61 \%$ & 217 & $30.61 \%$ & \multirow{2}{*}{0.984} \\
\hline$<65$ & 3329 & $64.55 \%$ & 1513 & $64.77 \%$ & & 492 & $69.39 \%$ & 492 & $69.39 \%$ & \\
\hline \multicolumn{11}{|l|}{ Insurance } \\
\hline Uninsured & 608 & $11.79 \%$ & 207 & $8.86 \%$ & \multirow{3}{*}{$<0.001$} & 16 & $2.26 \%$ & 16 & $2.26 \%$ & \multirow{3}{*}{1.000} \\
\hline Insured & 2980 & $57.79 \%$ & 1489 & $63.74 \%$ & & 532 & $75.04 \%$ & 532 & $75.04 \%$ & \\
\hline Unknown & 1569 & $30.42 \%$ & 640 & $27.40 \%$ & & 161 & $22.71 \%$ & 161 & $22.71 \%$ & \\
\hline \multicolumn{11}{|l|}{ Tumor Grade } \\
\hline 1 & 265 & $5.14 \%$ & 117 & $5.01 \%$ & \multirow{4}{*}{$<0.001$} & 23 & $3.24 \%$ & 23 & $3.24 \%$ & \multirow{4}{*}{1.000} \\
\hline 2 & 1744 & $33.82 \%$ & 886 & $37.93 \%$ & & 247 & $34.84 \%$ & 247 & $34.84 \%$ & \\
\hline 3 & 3090 & $59.92 \%$ & 1300 & $55.65 \%$ & & 436 & $61.50 \%$ & 436 & $61.50 \%$ & \\
\hline 4 & 58 & $1.12 \%$ & 33 & $1.41 \%$ & & 3 & $0.42 \%$ & 3 & $0.42 \%$ & \\
\hline \multicolumn{11}{|l|}{ Race } \\
\hline Black & 801 & $15.53 \%$ & 201 & $8.60 \%$ & \multirow{4}{*}{$<0.001$} & 11 & $1.55 \%$ & 11 & $1.55 \%$ & \multirow{4}{*}{1.000} \\
\hline other & 597 & $11.58 \%$ & 174 & $7.45 \%$ & & 14 & $1.97 \%$ & 14 & $1.97 \%$ & \\
\hline White & 3743 & $72.58 \%$ & 1957 & $83.78 \%$ & & 684 & $96.47 \%$ & 684 & $96.47 \%$ & \\
\hline unknown & 16 & $0.31 \%$ & 4 & $0.17 \%$ & & 0 & $0.00 \%$ & 0 & $0.00 \%$ & \\
\hline \multicolumn{11}{|l|}{ Marital Status } \\
\hline Divorced & 429 & $8.32 \%$ & 206 & $8.82 \%$ & \multirow{6}{*}{$<0.001$} & 29 & $4.09 \%$ & 29 & $4.09 \%$ & \multirow{6}{*}{1.000} \\
\hline Married & 2657 & $51.52 \%$ & 1447 & $61.94 \%$ & & 537 & $75.74 \%$ & 537 & $75.74 \%$ & \\
\hline Separated & 57 & $1.11 \%$ & 21 & $0.90 \%$ & & 0 & $0.00 \%$ & 0 & $0.00 \%$ & \\
\hline Single & 739 & $14.33 \%$ & 252 & $10.79 \%$ & & 47 & $6.63 \%$ & 47 & $6.63 \%$ & \\
\hline Widowed & 259 & $5.02 \%$ & 75 & $3.21 \%$ & & 10 & $1.41 \%$ & 10 & $1.41 \%$ & \\
\hline unknown & 1016 & $19.70 \%$ & 335 & $14.34 \%$ & & 86 & $12.13 \%$ & 86 & $12.13 \%$ & \\
\hline Primary Site & & & & & & & & & & \\
\hline Cardia & 1909 & $37.02 \%$ & 1785 & $76.41 \%$ & & 611 & $86.18 \%$ & 611 & $86.18 \%$ & \\
\hline Fundus of Stomach & 246 & $4.77 \%$ & 56 & $2.40 \%$ & & 5 & $0.71 \%$ & 5 & $0.71 \%$ & \\
\hline Body of Stomach & 459 & $8.90 \%$ & 72 & $3.08 \%$ & & 16 & $2.26 \%$ & 16 & $2.26 \%$ & \\
\hline Gastric Antrum & 836 & $16.21 \%$ & 125 & $5.35 \%$ & & 34 & $4.80 \%$ & 34 & $4.80 \%$ & \\
\hline Pylorus & 109 & $2.11 \%$ & 14 & $0.60 \%$ & $<0.001$ & 0 & $0.00 \%$ & 0 & $0.00 \%$ & 1.000 \\
\hline Lesser Curvature of Stomach & 344 & $6.67 \%$ & 61 & $2.61 \%$ & & 4 & $0.56 \%$ & 4 & $0.56 \%$ & \\
\hline Greater Curvature of Stomach & 153 & $2.97 \%$ & 26 & $1.11 \%$ & & 2 & $0.28 \%$ & 2 & $0.28 \%$ & \\
\hline Overlapping Lesion of Stomach & 387 & $7.50 \%$ & 86 & $3.68 \%$ & & 8 & $1.13 \%$ & 8 & $1.13 \%$ & \\
\hline Stomach & 714 & $13.85 \%$ & 111 & $4.75 \%$ & & 29 & $4.09 \%$ & 29 & $4.09 \%$ & \\
\hline T Stage (AJCC, 2004) & & & & & & & & & & \\
\hline TO & 6 & $0.12 \%$ & 1 & $0.04 \%$ & & 0 & $0.00 \%$ & 0 & $0.00 \%$ & \\
\hline T1 & 2633 & $51.06 \%$ & 774 & $33.13 \%$ & & 379 & $53.46 \%$ & 379 & $53.46 \%$ & \\
\hline T2a & 318 & $6.17 \%$ & 264 & $11.30 \%$ & $<0 \Omega 1$ & 45 & $6.35 \%$ & 45 & $6.35 \%$ & 1 \\
\hline $\mathrm{T} 2 \mathrm{~b}$ & 667 & $12.93 \%$ & 581 & $24.87 \%$ & $<0.001$ & 123 & $17.35 \%$ & 123 & $17.35 \%$ & 1.000 \\
\hline T3 & 320 & $6.21 \%$ & 350 & $14.98 \%$ & & 69 & $9.73 \%$ & 69 & $9.73 \%$ & \\
\hline T4 & 1213 & $23.52 \%$ & 366 & $15.67 \%$ & & 93 & $13.12 \%$ & 93 & $13.12 \%$ & \\
\hline
\end{tabular}




\begin{tabular}{|c|c|c|c|c|c|c|c|c|c|c|}
\hline N Stac & & & & & & & & & & \\
\hline NO & 3168 & $61.43 \%$ & 1093 & $46.79 \%$ & \multirow{4}{*}{$<0.001$} & 389 & $54.87 \%$ & 389 & $54.87 \%$ & \multirow{4}{*}{0.998} \\
\hline N1 & 1788 & $34.67 \%$ & 1085 & $46.45 \%$ & & 306 & $43.16 \%$ & 306 & $43.16 \%$ & \\
\hline N2 & 146 & $2.83 \%$ & 128 & $5.48 \%$ & & 13 & $1.83 \%$ & 13 & $1.83 \%$ & \\
\hline N3 & 55 & $1.07 \%$ & 30 & $1.28 \%$ & & 1 & $0.14 \%$ & 1 & $0.14 \%$ & \\
\hline \multicolumn{11}{|c|}{ M Stage (AJCC, 2004) } \\
\hline M1 & 2715 & $52.65 \%$ & 808 & $34.59 \%$ & $<0.001$ & 298 & $42.03 \%$ & 298 & $42.03 \%$ & 1.000 \\
\hline \multicolumn{11}{|c|}{ Tumor Size } \\
\hline$\leq 1 \mathrm{~cm}$ & 2002 & $38.82 \%$ & 1238 & $53.00 \%$ & \multirow{2}{*}{$<0.001$} & 352 & $49.65 \%$ & 352 & $49.65 \%$ & \multirow{2}{*}{1.000} \\
\hline$>1 \mathrm{~cm}$ & 3155 & $61.18 \%$ & 1098 & $47.00 \%$ & & 357 & $50.35 \%$ & 357 & $50.35 \%$ & \\
\hline
\end{tabular}

Table 2: Demographics according to radiotherapy vs. non-radiotherapy for SRCC patients without gastrectomy.

\begin{tabular}{|c|c|c|c|c|c|c|c|c|c|c|}
\hline & \multicolumn{5}{|c|}{ Unadjusted } & \multicolumn{5}{|c|}{ Adjusted } \\
\hline & Non-rad & SD/\% & Rad & SD $/ \%$ & $\mathbf{p}$ & Non-rad & SD/\% & Rad & SD/\% & $\mathbf{p}$ \\
\hline No. & 1705 & $79.23 \%$ & 447 & $20.77 \%$ & - & 81 & $50 \%$ & 81 & $50 \%$ & - \\
\hline \multicolumn{11}{|l|}{ Gender } \\
\hline Male & 864 & $50.67 \%$ & 1784 & $50.40 \%$ & \multirow{2}{*}{$<0.001$} & 60 & $74.07 \%$ & 60 & $74.07 \%$ & \multirow{2}{*}{1.000} \\
\hline Female & 841 & $49.33 \%$ & 1756 & $49.60 \%$ & & 21 & $25.93 \%$ & 21 & $25.93 \%$ & \\
\hline \multicolumn{11}{|l|}{ Age (yrs) } \\
\hline$\geq 65$ & 918 & $53.84 \%$ & 1858 & $52.49 \%$ & \multirow{2}{*}{$<0.001$} & 32 & $39.51 \%$ & 32 & $39.51 \%$ & \multirow{2}{*}{0.980} \\
\hline$<65$ & 787 & $46.16 \%$ & 1682 & $47.51 \%$ & & 49 & $60.49 \%$ & 49 & $60.49 \%$ & \\
\hline \multicolumn{11}{|l|}{ Insurance } \\
\hline Uninsured & 242 & $14.19 \%$ & 523 & $14.77 \%$ & \multirow[b]{2}{*}{$<0.001$} & 2 & $2.47 \%$ & 2 & $2.47 \%$ & \multirow[b]{2}{*}{1.000} \\
\hline Insured & 947 & $55.54 \%$ & 1785 & $50.42 \%$ & & 64 & $79.01 \%$ & 64 & $79.01 \%$ & \\
\hline \multicolumn{11}{|c|}{ Tumor Grade } \\
\hline 1 & 3 & $0.18 \%$ & 6 & $0.17 \%$ & \multirow{4}{*}{$<0.001$} & 0 & $0.00 \%$ & 0 & $0.00 \%$ & \multirow{4}{*}{1.000} \\
\hline 2 & 39 & $2.29 \%$ & 72 & $2.03 \%$ & & 0 & $0.00 \%$ & 0 & $0.00 \%$ & \\
\hline 3 & 1617 & $94.84 \%$ & 3378 & $95.42 \%$ & & 81 & $100 \%$ & 81 & $100 \%$ & \\
\hline 4 & 46 & $2.70 \%$ & 84 & $2.37 \%$ & & 0 & $0.00 \%$ & 0 & $0.00 \%$ & \\
\hline \multicolumn{11}{|l|}{ Race } \\
\hline Black & 254 & $14.90 \%$ & 443 & $12.51 \%$ & \multirow{4}{*}{$<0.001$} & 2 & $2.47 \%$ & 2 & $2.47 \%$ & \multirow{4}{*}{1.000} \\
\hline Other & 232 & $13.61 \%$ & 509 & $14.38 \%$ & & 5 & $6.17 \%$ & 5 & $6.17 \%$ & \\
\hline White & 1211 & $71.03 \%$ & 2564 & $72.43 \%$ & & 74 & $91.36 \%$ & 74 & $91.36 \%$ & \\
\hline Unknown & 8 & $0.47 \%$ & 24 & $0.68 \%$ & & 0 & $0.00 \%$ & 0 & $0.00 \%$ & \\
\hline \multicolumn{11}{|c|}{ Marital Status } \\
\hline Married & 899 & $52.73 \%$ & 1816 & $51.30 \%$ & \multirow{5}{*}{$<0.001$} & 62 & $76.54 \%$ & 62 & $76.54 \%$ & \multirow{5}{*}{1.000} \\
\hline Separated & 17 & $1.00 \%$ & 39 & $1.10 \%$ & & 0 & $0.00 \%$ & 0 & $0.00 \%$ & \\
\hline Single & 297 & $17.42 \%$ & 624 & $17.63 \%$ & & 6 & $7.41 \%$ & 6 & $7.41 \%$ & \\
\hline Widowed & 72 & $4.22 \%$ & 571 & $16.13 \%$ & & 1 & $1.23 \%$ & 1 & $1.23 \%$ & \\
\hline Unknown & 264 & $15.48 \%$ & 201 & $5.68 \%$ & & 12 & $14.81 \%$ & 12 & $14.81 \%$ & \\
\hline Primary Site & & & & & & & & & & \\
\hline
\end{tabular}




\begin{tabular}{|c|c|c|c|c|c|c|c|c|c|c|}
\hline Cardia & 296 & $17.36 \%$ & 576 & $16.27 \%$ & \multirow{9}{*}{$<0.001$} & 47 & $50.72 \%$ & 47 & $50.72 \%$ & \multirow{9}{*}{1.000} \\
\hline Fundus of Stomach & 73 & $4.28 \%$ & 144 & $4.07 \%$ & & 2 & $1.45 \%$ & 2 & $1.45 \%$ & \\
\hline Body of Stomach & 229 & $13.43 \%$ & 505 & $14.27 \%$ & & 5 & $5.80 \%$ & 5 & $5.80 \%$ & \\
\hline Gastric Antrum & 341 & $20.00 \%$ & 655 & $18.50 \%$ & & 7 & $10.87 \%$ & 7 & $10.87 \%$ & \\
\hline Pylorus & 33 & $1.94 \%$ & 71 & $2.01 \%$ & & 1 & $0.72 \%$ & 1 & $0.72 \%$ & \\
\hline Lesser Curvature of Stomach & 98 & $5.75 \%$ & 199 & $5.62 \%$ & & 2 & $2.90 \%$ & 2 & $2.90 \%$ & \\
\hline Greater Curvature of Stomach & 69 & $4.05 \%$ & 127 & $3.59 \%$ & & 2 & $1.45 \%$ & 2 & $1.45 \%$ & \\
\hline Overlapping Lesion of Stomach & 238 & $13.96 \%$ & 424 & $11.98 \%$ & & 5 & $5.07 \%$ & 5 & $5.07 \%$ & \\
\hline Stomach & 328 & $19.24 \%$ & 839 & $23.70 \%$ & & 10 & $21.01 \%$ & 10 & $21.01 \%$ & \\
\hline \multicolumn{11}{|l|}{ T Stage (AJCC, 2004) } \\
\hline TO & 5 & $0.29 \%$ & 6 & $0.17 \%$ & \multirow{6}{*}{$<0.001$} & 0 & $0.00 \%$ & 0 & $0.00 \%$ & \multirow{6}{*}{1.000} \\
\hline $\mathrm{T} 1$ & 622 & $36.48 \%$ & 725 & $20.48 \%$ & & 40 & $49.38 \%$ & 40 & $49.38 \%$ & \\
\hline $\mathrm{T} 2 \mathrm{a}$ & 183 & $10.73 \%$ & 235 & $6.64 \%$ & & 2 & $2.47 \%$ & 2 & $2.47 \%$ & \\
\hline $\mathrm{T} 2 \mathrm{~b}$ & 245 & $14.37 \%$ & 288 & $8.14 \%$ & & 14 & $17.28 \%$ & 14 & $17.28 \%$ & \\
\hline T3 & 159 & $9.33 \%$ & 195 & $5.51 \%$ & & 5 & $6.17 \%$ & 5 & $6.17 \%$ & \\
\hline T4 & 491 & $28.80 \%$ & 650 & $18.36 \%$ & & 20 & $24.69 \%$ & 20 & $24.69 \%$ & \\
\hline \multicolumn{11}{|l|}{ N Stage (AJCC, 2004) } \\
\hline NO & 1089 & $63.87 \%$ & 1562 & $44.12 \%$ & \multirow{4}{*}{$<0.001$} & 49 & $60.49 \%$ & 49 & $60.49 \%$ & \multirow{4}{*}{1.000} \\
\hline N1 & 553 & $32.43 \%$ & 784 & $22.15 \%$ & & 32 & $39.51 \%$ & 32 & $39.51 \%$ & \\
\hline N2 & 33 & $1.94 \%$ & 41 & $1.16 \%$ & & 0 & $0.00 \%$ & 0 & $0.00 \%$ & \\
\hline N3 & 30 & $1.76 \%$ & 34 & $0.96 \%$ & & 0 & $0.00 \%$ & 0 & $0.00 \%$ & \\
\hline \multicolumn{11}{|l|}{ M Stage (AJCC, 2004) } \\
\hline MO & 693 & $40.65 \%$ & 986 & $27.85 \%$ & \multirow{2}{*}{$<0.001$} & 42 & $51.85 \%$ & 42 & $51.85 \%$ & \multirow{2}{*}{1.000} \\
\hline M1 & 2101 & $59.35 \%$ & 2139 & $60.42 \%$ & & 39 & $48.15 \%$ & 39 & $48.15 \%$ & \\
\hline \multicolumn{11}{|l|}{ Tumor Size } \\
\hline$\leq 1 \mathrm{~cm}$ & 437 & $25.63 \%$ & 729 & $20.59 \%$ & \multirow{2}{*}{$<0.001$} & 24 & $29.63 \%$ & 24 & $29.63 \%$ & \multirow{2}{*}{1.000} \\
\hline$>1 \mathrm{~cm}$ & 1268 & $74.37 \%$ & 2811 & $79.41 \%$ & & 57 & $70.37 \%$ & 57 & $70.37 \%$ & \\
\hline
\end{tabular}

Table 3: Demographics according to radiotherapy vs. non-radiotherapy for NSRCC patients with gastrectomy.

\begin{tabular}{|c|c|c|c|c|c|c|c|c|c|c|}
\hline & \multicolumn{5}{|c|}{ Unadjusted } & \multicolumn{5}{|c|}{ Adjusted } \\
\hline & Non-rad & $\mathrm{SD} / \%$ & $\operatorname{Rad}$ & $\mathrm{SD} / \%$ & $\mathbf{p}$ & Non-rad & SD $/ \%$ & Rad & SD $/ \%$ & $p$ \\
\hline No. & 6804 & $66.79 \%$ & 3382 & $33.21 \%$ & - & 132 & $50 \%$ & 132 & $50 \%$ & - \\
\hline \multicolumn{11}{|l|}{ Gender } \\
\hline Male & 4447 & $65.36 \%$ & 2541 & $75.13 \%$ & \multirow{2}{*}{$<0.001$} & 124 & $93.94 \%$ & 124 & $93.94 \%$ & \multirow{2}{*}{0.990} \\
\hline Female & 2357 & $34.64 \%$ & 841 & $24.87 \%$ & & 8 & $6.06 \%$ & 8 & $6.06 \%$ & \\
\hline \multicolumn{11}{|l|}{ Age (yrs) } \\
\hline$\geq 65$ & 2160 & $31.75 \%$ & 1888 & $55.82 \%$ & \multirow{2}{*}{$<0.001$} & & $31.06 \%$ & 41 & $31.06 \%$ & \multirow{2}{*}{0.984} \\
\hline$<65$ & 4644 & $68.25 \%$ & 1494 & $44.18 \%$ & & 91 & $68.94 \%$ & 91 & $68.94 \%$ & \\
\hline \multicolumn{11}{|l|}{ Insurance } \\
\hline Uninsured & 694 & $10.20 \%$ & 278 & $8.22 \%$ & \multirow{3}{*}{$<0.001$} & 0 & $0.00 \%$ & 0 & $0.00 \%$ & \multirow{3}{*}{1.000} \\
\hline Insured & 3563 & $52.37 \%$ & 1937 & $57.27 \%$ & & 112 & $84.85 \%$ & 112 & $84.85 \%$ & \\
\hline Unknown & 2547 & $37.43 \%$ & 1167 & $34.51 \%$ & & 20 & $15.15 \%$ & 20 & $15.15 \%$ & \\
\hline \multicolumn{11}{|c|}{ Tumor Grade } \\
\hline 1 & 509 & $7.48 \%$ & 123 & $3.64 \%$ & \multirow{4}{*}{$<0.001$} & 4 & $3.03 \%$ & 4 & $3.03 \%$ & \multirow{3}{*}{1.000} \\
\hline 2 & 2378 & $34.95 \%$ & 1109 & $32.79 \%$ & & 54 & $40.91 \%$ & 54 & $40.91 \%$ & \\
\hline 3 & 3789 & $55.69 \%$ & 2073 & $61.30 \%$ & & 74 & $56.06 \%$ & 74 & $56.06 \%$ & \\
\hline 4 & 128 & $1.88 \%$ & 77 & $2.28 \%$ & & 0 & $0.00 \%$ & 0 & $0.00 \%$ & \\
\hline
\end{tabular}




\begin{tabular}{|c|c|c|c|c|c|c|c|c|c|c|}
\hline Race & & & & & & & & & & \\
\hline Black & 909 & $13.36 \%$ & 369 & $10.91 \%$ & \multirow{4}{*}{$<0.001$} & 0 & $0.00 \%$ & 0 & $0.00 \%$ & \multirow{4}{*}{1.000} \\
\hline other & 1202 & $17.67 \%$ & 500 & $14.78 \%$ & & 0 & $0.00 \%$ & 0 & $0.00 \%$ & \\
\hline White & 4671 & $68.65 \%$ & 2508 & $74.16 \%$ & & 0 & $0.00 \%$ & 0 & $0.00 \%$ & \\
\hline unknown & 22 & $0.32 \%$ & 5 & $0.15 \%$ & & 132 & $100.00 \%$ & 132 & $100.00 \%$ & \\
\hline \multicolumn{11}{|l|}{ Marital Status } \\
\hline Divorced & 45 & $0.66 \%$ & 277 & $8.19 \%$ & \multirow{6}{*}{$<0.001$} & 1 & $0.76 \%$ & 1 & $0.76 \%$ & \multirow{6}{*}{1.000} \\
\hline Married & 4068 & $59.79 \%$ & 2334 & $69.01 \%$ & & 121 & $91.67 \%$ & 121 & $91.67 \%$ & \\
\hline Separated & 73 & $1.07 \%$ & 34 & $1.01 \%$ & & 0 & $0.00 \%$ & 0 & $0.00 \%$ & \\
\hline Single & 785 & $11.54 \%$ & 369 & $10.91 \%$ & & 3 & $2.27 \%$ & 3 & $2.27 \%$ & \\
\hline Widowed & 228 & $3.35 \%$ & 89 & $2.63 \%$ & & 0 & $0.00 \%$ & 0 & $0.00 \%$ & \\
\hline Unknown & 1175 & $17.27 \%$ & 279 & $8.25 \%$ & & 7 & $5.30 \%$ & 7 & $5.30 \%$ & \\
\hline \multicolumn{11}{|l|}{ Primary Site } \\
\hline Cardia & 1951 & $28.67 \%$ & 1762 & $52.10 \%$ & \multirow{9}{*}{$<0.001$} & 131 & $99.24 \%$ & 131 & $99.24 \%$ & \multirow{9}{*}{1.000} \\
\hline Fundus of Stomach & 191 & $2.81 \%$ & 81 & $2.40 \%$ & & 0 & $0.00 \%$ & 0 & $0.00 \%$ & \\
\hline Body of Stomach & 541 & $7.95 \%$ & 176 & $5.20 \%$ & & 0 & $0.00 \%$ & 0 & $0.00 \%$ & \\
\hline Gastric Antrum & 1734 & $25.49 \%$ & 531 & $15.70 \%$ & & 0 & $0.00 \%$ & 0 & $0.00 \%$ & \\
\hline Pylorus & 308 & $4.53 \%$ & 117 & $3.46 \%$ & & 0 & $0.00 \%$ & 0 & $0.00 \%$ & \\
\hline Lesser Curvature of Stomach & 741 & $10.89 \%$ & 271 & $8.01 \%$ & & 0 & $0.00 \%$ & 0 & $0.00 \%$ & \\
\hline Greater Curvature of Stomach & 326 & $4.79 \%$ & 125 & $3.70 \%$ & & 0 & $0.00 \%$ & 0 & $0.00 \%$ & \\
\hline Overlapping Lesion of Stomach & 368 & $5.41 \%$ & 140 & $4.14 \%$ & & 0 & $0.00 \%$ & 0 & $0.00 \%$ & \\
\hline Stomach & 644 & $9.47 \%$ & 179 & $5.29 \%$ & & 1 & $0.76 \%$ & 1 & $0.76 \%$ & \\
\hline \multicolumn{11}{|l|}{ T Stage (AJCC, 2004) } \\
\hline T0 & 0 & $0.00 \%$ & 0 & $0.00 \%$ & \multirow{6}{*}{$<0.001$} & 0 & $0.00 \%$ & 0 & $0.00 \%$ & \multirow{6}{*}{1.000} \\
\hline T1 & 1981 & $29.12 \%$ & 287 & $8.49 \%$ & & 46 & $34.85 \%$ & 46 & $34.85 \%$ & \\
\hline $\mathrm{T} 2 \mathrm{a}$ & 911 & $13.39 \%$ & 442 & $13.07 \%$ & & 16 & $12.12 \%$ & 16 & $12.12 \%$ & \\
\hline $\mathrm{T} 2 \mathrm{~b}$ & 2083 & $30.61 \%$ & 1429 & $42.25 \%$ & & 40 & $30.30 \%$ & 40 & $30.30 \%$ & \\
\hline T3 & 1194 & $17.55 \%$ & 956 & $28.27 \%$ & & 26 & $19.70 \%$ & 26 & $19.70 \%$ & \\
\hline $\mathrm{T} 4$ & 635 & $9.33 \%$ & 268 & $7.92 \%$ & & 4 & $3.03 \%$ & 4 & $3.03 \%$ & \\
\hline \multicolumn{11}{|l|}{ N Stage (AJCC, 2004) } \\
\hline NO & 3337 & $49.04 \%$ & 818 & $24.19 \%$ & \multirow{4}{*}{$<0.001$} & 61 & $46.21 \%$ & 61 & $46.21 \%$ & \multirow{4}{*}{0.998} \\
\hline N1 & 2223 & $32.67 \%$ & 1833 & $54.20 \%$ & & 67 & $50.76 \%$ & 67 & $50.76 \%$ & \\
\hline N2 & 849 & $12.48 \%$ & 562 & $16.62 \%$ & & 4 & $3.03 \%$ & 4 & $3.03 \%$ & \\
\hline N3 & 395 & $5.81 \%$ & 169 & $5.00 \%$ & & 0 & $0.00 \%$ & 0 & $0.00 \%$ & \\
\hline \multicolumn{11}{|l|}{ M Stage (AJCC, 2004) } \\
\hline MO & 5873 & $86.32 \%$ & 3168 & $93.67 \%$ & \multirow{2}{*}{$<0.001$} & 119 & $90.15 \%$ & 119 & $90.15 \%$ & \multirow{2}{*}{1.000} \\
\hline M1 & 931 & $13.68 \%$ & 214 & $6.33 \%$ & & 13 & $9.85 \%$ & 13 & $9.85 \%$ & \\
\hline \multicolumn{11}{|l|}{ Tumor Size } \\
\hline$\leq 1 \mathrm{~cm}$ & 6035 & $88.70 \%$ & 2895 & $85.60 \%$ & \multirow{2}{*}{$<0.001$} & 99 & $75.00 \%$ & 99 & $75.00 \%$ & \multirow{2}{*}{1.000} \\
\hline$>1 \mathrm{~cm}$ & 769 & $11.30 \%$ & 487 & $14.40 \%$ & & 33 & $25.00 \%$ & 33 & $25.00 \%$ & \\
\hline
\end{tabular}

SRCC patients (HR, 1.501; 95\% CI, 1.405-1.602; $\mathrm{p}<0.001)$ is better than that for NSRCC patients (HR, 1.356; 95\% CI, 1.304-1.410; $\mathrm{p}<0.001)$. But from the multivariate analysis data, we can know that radiotherapy for SRCC (HR, 1.352; 95\% CI, 1.258-1.453; $<<0.001)$ and NSRCC (HR, 1.405; 95\% CI, 1.347-1.466; $\mathrm{p}<0.001)$ is not far apart.

The univariate analysis of CSS time show that radiotherapy has also better effects on SRCC patients (HR, 1.413; 95\% CI, 1.3111.523; $\mathrm{p}<0.001)$ than NSRCC patients $(\mathrm{HR}, 1.250$; $95 \% \mathrm{CI}, 1.193$ $1.309 ; \mathrm{p}<0.001)$. From the multivariate analysis, we can also know radiotherapy for SRCC (HR, 1.254; 95\% CI, 1.155-1.361; $\mathrm{p}<0.001)$ and NSRCC (HR, 1.326; 95\% CI, 1.260-1.395; p<0.001) is also not far apart. 
Table 4: Demographics according to radiotherapy vs. non-radiotherapy for SRCC patients with gastrectomy.

\begin{tabular}{|c|c|c|c|c|c|c|c|c|c|c|}
\hline & \multicolumn{5}{|c|}{ Unadjusted } & \multicolumn{5}{|c|}{ Adjusted } \\
\hline & Non-rad & SD/\% & Rad & $\mathrm{SD} / \%$ & $\mathbf{p}$ & Non-rad & SD/\% & Rad & $\mathrm{SD} / \%$ & $\mathbf{p}$ \\
\hline No. & 2720 & 0.657 & 1420 & 0.343 & - & 291 & 0.5 & 291 & 0.5 & - \\
\hline \multicolumn{11}{|l|}{ Gender } \\
\hline Male & 1377 & 0.5063 & 773 & 0.5425 & \multirow{2}{*}{$<0.001$} & 186 & 0.6392 & 186 & 0.6392 & \multirow{2}{*}{1.000} \\
\hline Female & 1343 & 0.4938 & 652 & 0.4575 & & 105 & 0.3608 & 105 & 0.3608 & \\
\hline \multicolumn{11}{|l|}{ Age (yrs) } \\
\hline$\geq 65$ & 1400 & 0.5147 & 944 & 0.6625 & \multirow{2}{*}{$<0.001$} & 182 & 0.6254 & 182 & 0.6254 & \multirow{2}{*}{0.984} \\
\hline$<65$ & 1320 & 0.4853 & 481 & 0.3375 & & 109 & 0.3746 & 109 & 0.3746 & \\
\hline \multicolumn{11}{|l|}{ Insurance } \\
\hline Uninsured & 293 & 0.1077 & 146 & 0.1025 & \multirow{3}{*}{$<0.001$} & 11 & 0.0378 & 11 & 0.0378 & \multirow{3}{*}{1.000} \\
\hline Insured & 1431 & 0.5261 & 742 & 0.5207 & & 193 & 0.6632 & 193 & 0.6632 & \\
\hline Unknown & 996 & 0.3662 & 537 & 0.3768 & & 87 & 0.299 & 87 & 0.299 & \\
\hline \multicolumn{11}{|l|}{ Tumor Grade } \\
\hline 1 & 7 & 0.0026 & 6 & 0.0042 & \multirow{4}{*}{$<0.001$} & 0 & 0 & 0 & 0 & \multirow{4}{*}{1.000} \\
\hline 2 & 78 & 0.0287 & 38 & 0.0267 & & 2 & 0.0069 & 2 & 0.0069 & \\
\hline 3 & 2532 & 0.9309 & 1324 & 0.9291 & & 287 & 0.9863 & 287 & 0.9863 & \\
\hline 4 & 103 & 0.0379 & 57 & 0.04 & & 2 & 0.0069 & 2 & 0.0069 & \\
\hline \multicolumn{11}{|l|}{ Race } \\
\hline Black & 307 & 0.1129 & 177 & 0.1242 & \multirow{4}{*}{$<0.001$} & 9 & 0.0309 & 9 & 0.0309 & \multirow{4}{*}{1.000} \\
\hline other & 526 & 0.1934 & 281 & 0.1972 & & 39 & 0.134 & 39 & 0.134 & \\
\hline White & 1877 & 0.6901 & 961 & 0.6744 & & 243 & 0.8351 & 243 & 0.8351 & \\
\hline unknown & 10 & 0.0037 & 6 & 0.0042 & & 0 & 0 & 0 & 0 & \\
\hline \multicolumn{11}{|l|}{ Marital Status } \\
\hline Divorced & 213 & 0.0783 & 117 & 0.0821 & \multirow{6}{*}{$<0.001$} & 7 & 0.0241 & 7 & 0.0241 & \multirow{6}{*}{1.000} \\
\hline Married & 1639 & 0.6026 & 938 & 0.6582 & & 247 & 0.8488 & 247 & 0.8488 & \\
\hline Separated & 30 & 0.011 & 17 & 0.0119 & & 0 & 0 & 0 & 0 & \\
\hline Single & 383 & 0.1408 & 195 & 0.1368 & & 18 & 0.0619 & 18 & 0.0619 & \\
\hline Widowed & 101 & 0.0371 & 45 & 0.0316 & & 3 & 0.0103 & 3 & 0.0103 & \\
\hline unknown & 354 & 0.1301 & 113 & 0.0793 & & 16 & 0.055 & 16 & 0.055 & \\
\hline Primary Site & & & & & & & & & & \\
\hline Cardia & 298 & 0.1096 & 295 & 0.207 & & 72 & 0.2474 & 72 & 0.2474 & \\
\hline Fundus of stomach & 69 & 0.0254 & 40 & 0.0281 & & 3 & 0.0103 & 3 & 0.0103 & \\
\hline Body of stomach & 312 & 0.1147 & 132 & 0.0926 & & 30 & 0.1031 & 30 & 0.1031 & \\
\hline Gastric antrum & 784 & 0.2882 & 386 & 0.2709 & & 105 & 0.3608 & 105 & 0.3608 & \\
\hline Pylorus & 110 & 0.0404 & 66 & 0.0463 & $<0.001$ & 1 & 0.0034 & 1 & 0.0034 & 1.000 \\
\hline Lesser curvature of stomach & 350 & 0.1287 & 180 & 0.1263 & & 34 & 0.1168 & 34 & 0.1168 & \\
\hline Greater curvature of stomach & 172 & 0.0632 & 69 & 0.0484 & & 6 & 0.0206 & 6 & 0.0206 & \\
\hline Overlapping lesion of stomach & 315 & 0.1158 & 131 & 0.0919 & & 28 & 0.0962 & 28 & 0.0962 & \\
\hline stomach & 310 & 0.114 & 126 & 0.0884 & & 12 & 0.0412 & 12 & 0.0412 & \\
\hline T Stage (AJCC, 2004) & & & & & & & & & & \\
\hline T0 & 662 & 0.2434 & 0 & 0 & & 0 & 0 & 0 & 0 & \\
\hline T1 & 217 & 0.0798 & 97 & 0.0681 & & 25 & 0.0859 & 25 & 0.0859 & \\
\hline T2a & 731 & 0.2688 & 150 & 0.1053 & : & 22 & 0.0756 & 22 & 0.0756 & 1 \\
\hline $\mathrm{T} 2 \mathrm{~b}$ & 747 & 0.2746 & 558 & 0.3916 & - & 127 & 0.4364 & 127 & 0.4364 & 1.000 \\
\hline T3 & 338 & 0.1243 & 477 & 0.3347 & & 104 & 0.3574 & 104 & 0.3574 & \\
\hline T4 & 25 & 0.0092 & 143 & 0.1004 & & 13 & 0.0447 & 13 & 0.0447 & \\
\hline
\end{tabular}




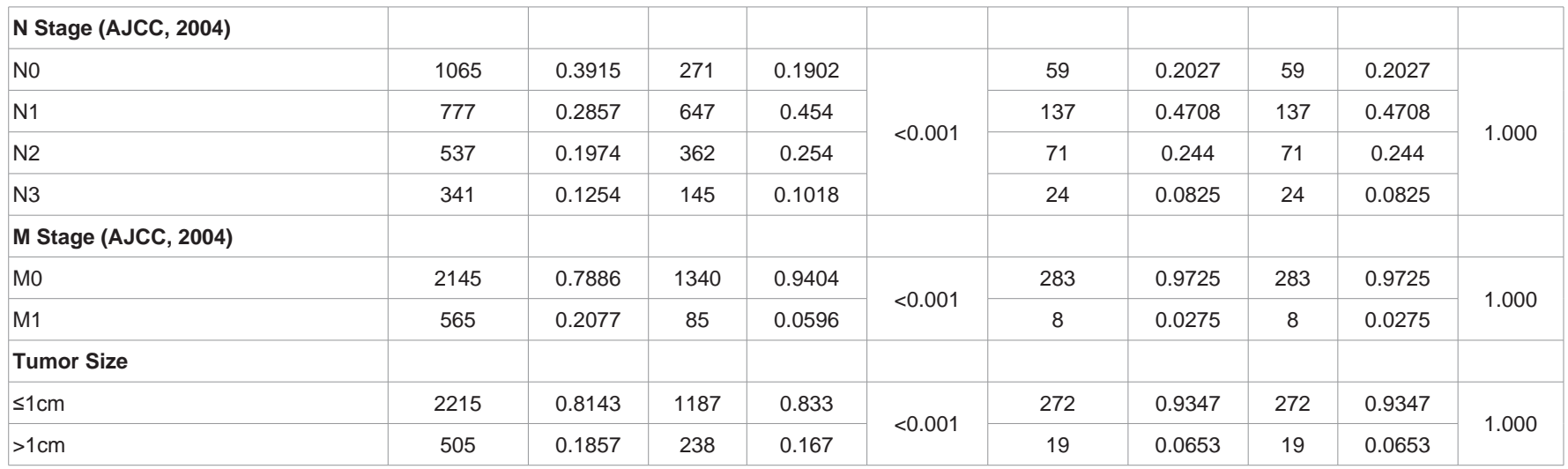

Table 5: Univariate and Multivariate Cox Analyses of SRCC Patients According to Various Clinic pathological Variables.

\begin{tabular}{|c|c|c|c|c|c|c|c|c|}
\hline \multirow{3}{*}{ Variable } & \multicolumn{4}{|c|}{ Overall Survival } & \multicolumn{4}{|c|}{ Cancer-Specific Survival } \\
\hline & \multicolumn{2}{|l|}{ Univariate } & \multicolumn{2}{|l|}{ Multivariate } & \multicolumn{2}{|l|}{ Univariate } & \multicolumn{2}{|l|}{ Multivariate } \\
\hline & HR $(95 \% \mathrm{Cl})$ & $\mathbf{P}$ & HR $(95 \% \mathrm{Cl})$ & $\mathbf{P}$ & HR $(95 \% \mathrm{Cl})$ & $\mathbf{P}$ & HR (95\%Cl) & $\mathbf{P}$ \\
\hline Gender & & 0.251 & & & & 0.94 & & \\
\hline Male & Reference & & - & & Reference & & - & \\
\hline Female & $1.023(0.984-1.063)$ & 0.294 & - & - & $0.997(0.931-1.068)$ & 0.94 & - & - \\
\hline Age & & $<0.001$ & & & & 0.201 & & \\
\hline$<65$ & Reference & & Reference & & Reference & & - & \\
\hline$\geq 65$ & $1.358(1.280-1.441)$ & $<0.001$ & $1.515(1.454-1.578)$ & $<0.001$ & $1.046(0.976-1.121)$ & 0.201 & - & - \\
\hline Insurance & & 0.055 & & & & 0.054 & & \\
\hline Uninsured & Reference & & Reference & - & - & & - & \\
\hline Insured & $0.991(0.842-1.167)$ & 0.915 & $0.934(0.777-1.122)$ & - & - & 0.463 & - & - \\
\hline Tumor Grade & & 0.131 & & & & 0.071 & & \\
\hline 1 & Reference & & Reference & - & - & & - & \\
\hline 2 & $2.136(1.044-4.369)$ & 0.038 & $1.854(0.808-4.251)$ & - & - & 0.145 & - & - \\
\hline 3 & $2.255(1.127-4.513)$ & 0.022 & $2.248(1.009-5.008)$ & - & - & 0.047 & - & - \\
\hline 4 & $2.278(1.120-4.635)$ & 0.023 & $2.343(1.032-5.316)$ & - & - & 0.042 & - & - \\
\hline Race & & $<0.001$ & & & & $<0.001$ & & \\
\hline Black & Reference & & Reference & & & & Reference & \\
\hline Other & $0.725(0.648-0.811)$ & $<0.001$ & $0.807(0.719-0.906)$ & $<0.001$ & $0.750(0.659-0.855)$ & $<0.001$ & $0.876(0.767-1.001)$ & 0.051 \\
\hline Unknown & $1.027(0.939-1.124)$ & 0.555 & $0.955(0.870-1.048)$ & 0.327 & $1.040(0.936-1.154)$ & 0.467 & $1.013(0.910-1.128)$ & 0.815 \\
\hline White & $0.397(0.188-0.836)$ & 0.015 & $0.428(0.203-0.904)$ & 0.026 & $0.457(0.204-1.023)$ & 0.057 & $0.524(0.234-1.176)$ & 0.117 \\
\hline Married & $0.926(0.830-1.034)$ & 0.174 & $0.917(0.820-1.025)$ & 0.126 & $0.965(0.849-1.098)$ & 0.59 & $0.976(0.858-1.111)$ & 0.714 \\
\hline Separated & $0.812(0.585-1.125)$ & 0.21 & $0.917(0.661-1.272)$ & 0.604 & $0.912(0.635-1.310)$ & 0.619 & $1.052(0.732-1.512)$ & 0.786 \\
\hline Single & $1.061(0.933-1.207)$ & 0.368 & $1.003(0.881-1.142)$ & 0.967 & $1.157(0.998-1.342)$ & 0.054 & $1.062(0.914-1.232)$ & 0.433 \\
\hline Widowed & $1.368(1.202-1.556)$ & $<0.001$ & $1.209(1.058-1.382)$ & 0.005 & $1.220(1.046-1.423)$ & 0.012 & $1.319(1.129-1.541)$ & $<0.001$ \\
\hline Other & $1.005(0.831-1.216)$ & 0.958 & $0.955(0.788-1.157)$ & 0.64 & $0.993(0.795-1.242)$ & 0.954 & $1.008(0.806-1.262)$ & 0.942 \\
\hline T Stage (AJCC, 2004) & & $<0.001$ & & & & $<0.001$ & & \\
\hline TO & Reference & & Reference & & Reference & & Reference & \\
\hline $\mathrm{T} 1$ & $0.260(0.117-0.581)$ & 0.001 & $0.665(0.295-1.495)$ & 0.323 & $0.267(0.100-0.715)$ & 0.009 & $0.725(0.269-1.954)$ & 0.525 \\
\hline T2a & $0.244(0.109-0.546)$ & 0.001 & $0.661(0.292-1.494)$ & 0.319 & $0.235(0.087-0.633)$ & 0.004 & $0.657(0.242-1.783)$ & 0.409 \\
\hline
\end{tabular}




\begin{tabular}{|c|c|c|c|c|c|c|c|c|}
\hline $\mathrm{T} 2 \mathrm{~b}$ & $0.312(0.140-0.696)$ & 0.004 & $0.881(0.391-1.984)$ & 0.76 & $0.359(0.134-0.958)$ & 0.041 & $1.010(0.374-2.725)$ & 0.984 \\
\hline T3 & $0.416(0.186-0.928)$ & 0.032 & $1.093(0.485-2.462)$ & 0.83 & $0.474(0.177-1.265)$ & 0.136 & $1.230(0.456-3.321)$ & 0.683 \\
\hline $\mathrm{T} 4$ & $0.681(0.305-1.521)$ & 0.349 & $1.170(0.520-2.633)$ & 0.704 & $0.833(0.312-2.226)$ & 0.716 & $1.346(0.499-3.628)$ & 0.557 \\
\hline N Stage (AJCC, 2004) & & $<0.001$ & & & & $<0.001$ & & \\
\hline NO & Reference & & Reference & & Reference & & Reference & \\
\hline N1 & $1.206(1.126-1.293)$ & $<0.001$ & $1.270(1.179-1.368)$ & $<0.001$ & $1.318(1.216-1.428)$ & $<0.001$ & $1.325(1.215-1.445)$ & $<0.001$ \\
\hline N2 & $1.297(1.189-1.414)$ & $<0.001$ & $1.804(1.632-1.993)$ & $<0.001$ & $1.442(1.305-1.593)$ & $<0.001$ & $1.979(1.764-2.220)$ & $<0.001$ \\
\hline N3 & $1.708(1.531-1.905)$ & $<0.001$ & $2.103(1.863-2.373)$ & $<0.001$ & $2.010(1.779-2.272)$ & $<0.001$ & $2.405(2.100-2.755)$ & $<0.001$ \\
\hline M Stage (AJCC, 2004) & & $<0.001$ & & & & $<0.001$ & & \\
\hline MO & Reference & & Reference & & Reference & & Reference & \\
\hline M1 & $3.111(2.916-3.319)$ & $<0.001$ & $1.701(1.577-1.834)$ & $<0.001$ & $3.647(3.389-3.925)$ & $<0.001$ & $1.844(1.693-2.009)$ & $<0.001$ \\
\hline Primary Site & & $<0.001$ & & & & $<0.001$ & & \\
\hline Fundus of Stomach & $0.961(0.805-1.146)$ & 0.655 & $0.998(0.835-1.194)$ & 0.985 & $1.035(0.849-1.261)$ & 0.733 & $1.094(0.896-1.337)$ & 0.378 \\
\hline Body of Stomach & $0.899(0.804-1.004)$ & 0.06 & $0.925(0.825-1.038)$ & 0.184 & $0.913(0.804-1.038)$ & 0.164 & $0.966(0.846-1.101)$ & 0.602 \\
\hline Gastric Antrum & $0.743(0.678-0.814)$ & $<0.001$ & $0.894(0.812-0.984)$ & 0.022 & $0.718(0.645-0.798)$ & $<0.001$ & $0.896(0.801-1.002)$ & 0.054 \\
\hline Pylorus & $0.686(0.575-0.819)$ & $<0.001$ & $0.828(0.691-0.992)$ & 0.041 & $0.687(0.559-0.844)$ & $<0.001$ & $0.869(0.704-1.072)$ & 0.189 \\
\hline Lesser Curvature of Stomach & $0.586(0.518-0.662)$ & $<0.001$ & $0.768(0.676-0.873)$ & $<0.001$ & $0.585(0.507-0.674)$ & $<0.001$ & $0.794(0.685-0.920)$ & 0.002 \\
\hline Greater Curvature of Stomach & $0.682(0.585-0.796)$ & $<0.001$ & $0.841(0.718-0.984)$ & 0.031 & $0.706(0.592-0.843)$ & $<0.001$ & $0.893(0.746-1.070)$ & 0.22 \\
\hline Overlapping Lesion of Stomach & $1.173(1.055-1.304)$ & 0.003 & $0.967(0.865-1.080)$ & 0.549 & $1.238(1.097-1.396)$ & 0.001 & $0.970(0.855-1.100)$ & 0.635 \\
\hline Stomach & $1.214(1.095-1.346)$ & $<0.001$ & $1.093(0.981-1.218)$ & 0.107 & $1.222(1.085-1.376)$ & 0.001 & $1.105(0.975-1.252)$ & 0.117 \\
\hline Tumor Size & & $<0.001$ & & & & $<0.001$ & & \\
\hline$\leq 1 \mathrm{~cm}$ & Reference & & Reference & & Reference & & & \\
\hline Yes & Reference & & Reference & & Reference & & Reference & \\
\hline No & $3.405(3.194-3.629)$ & $<0.001$ & $2.758(2.536-2.998)$ & $<0.001$ & $3.598(3.344-3.872)$ & $<0.001$ & $2.942(2.671-3.240)$ & $<0.001$ \\
\hline Radiation & & $<0.001$ & & & & $<0.001$ & & \\
\hline Yes & Reference & & & & Reference & & Reference & \\
\hline No & $1.501(1.405-1.602)$ & $<0.001$ & $1.352(1.258-1.453)$ & $<0.001$ & $1.413(1.311-1.523)$ & $<0.001$ & $1.254(1.155-1.361)$ & $<0.001$ \\
\hline
\end{tabular}

Table 6: Univariate and Multivariate Cox Analyses of NSRCC Patients According to Various Clinic pathological Variables.

\begin{tabular}{|c|c|c|c|c|c|c|c|c|}
\hline \multirow{3}{*}{ Variable } & \multicolumn{4}{|c|}{ Overall Survival } & \multicolumn{4}{|c|}{ Cancer-Specific Survival } \\
\hline & \multicolumn{2}{|l|}{ Univariate } & \multicolumn{2}{|c|}{ Multivariate } & \multicolumn{2}{|l|}{ Univariate } & \multicolumn{2}{|c|}{ Multivariate } \\
\hline & HR $(95 \% \mathrm{Cl})$ & $\mathbf{P}$ & HR $(95 \% \mathrm{Cl})$ & $\mathbf{P}$ & HR $(95 \% \mathrm{Cl})$ & $\mathbf{P}$ & HR $(95 \% \mathrm{Cl})$ & $\mathbf{P}$ \\
\hline Gender & & 0.251 & & & $<0.001$ & & & \\
\hline Male & Reference & & - & & Reference & - & - & \\
\hline Female & $1.023(0.984-1.063)$ & 0.251 & - & - & $1.041(0.994-1.091)$ & 0.09 & & - \\
\hline Age & & $<0.001$ & & & $<0.001$ & & & \\
\hline$<65$ & Reference & & Reference & & Reference & - & & \\
\hline$\geq 65$ & $1.382(1.331-1.436)$ & $<0.001$ & $1.515(1.454-1.578)$ & $<0.001$ & $1.036(0.991-1.083)$ & 0.123 & - & - \\
\hline Insurance & & 0.003 & & & $<0.001$ & & & \\
\hline Uninsured & Reference & & Reference & & Reference & & & \\
\hline Insured & $1.146(1.036-1.267)$ & 0.008 & $1.059(0.956-1.173)$ & 0.274 & $1.260(1.121-1.416)$ & $<0.001$ & $1.078(0.958-1.213)$ & 0.214 \\
\hline Unknown & $1.051(1.011-1.093)$ & 0.011 & $1.166(1.121-1.212)$ & $<0.001$ & $1.060(1.011-1.110)$ & 0.015 & $1.196(1.140-1.254)$ & $<0.001$ \\
\hline Tumor Grade & & $<0.001$ & & & & $<0.001$ & & \\
\hline
\end{tabular}




\begin{tabular}{|c|c|c|c|c|c|c|c|c|}
\hline 1 & Reference & & Reference & & Reference & & Reference & \\
\hline 2 & $1.439(1.312-1.577)$ & $<0.001$ & $1.258(1.146-1.380)$ & $<0.001$ & $1.664(1.470-1.884)$ & $<0.001$ & $1.344(1.186-1.523)$ & $<0.001$ \\
\hline 3 & $1.887(1.726-2.064)$ & $<0.001$ & $1.549(1.414-1.698)$ & $<0.001$ & $2.451(2.172-2.766)$ & $<0.001$ & $1.775(1.569-2.007)$ & $<0.001$ \\
\hline 4 & 1.702(1.446-2.005) & $<0.001$ & $1.481(1.255-1.746)$ & $<0.001$ & $2.196(1.792-2.691)$ & $<0.001$ & $1.693(1.379-2.078)$ & $<0.001$ \\
\hline Race & & $<0.001$ & & & & $<0.001$ & & \\
\hline Black & Reference & & Reference & & Reference & & Reference & \\
\hline Other & $0.675(0.630-0.725)$ & $<0.001$ & $0.765(0.712-0.821)$ & $<0.001$ & $0.734(0.675-0.799)$ & $<0.001$ & $0.848(0.779-0.924)$ & $<0.001$ \\
\hline Unknown & $0.512(0.326-0.804)$ & 0.004 & $0.919(0.870-0.970)$ & 0.002 & $0.884(0.830-0.943)$ & $<0.001$ & $0.926(0.866-0.990)$ & 0.025 \\
\hline White & $0.880(0.835-0.927)$ & $<0.001$ & $0.584(0.371-0.918)$ & 0.02 & $0.610(0.366-1.015)$ & 0.057 & $0.692(0.415-1.153)$ & 0.158 \\
\hline Marital Status & & $<0.001$ & & & & $<0.001$ & & \\
\hline Divorced & Reference & & Reference & & Reference & & Reference & \\
\hline Married & $0.832(0.777-0.890)$ & $<0.001$ & $0.853(0.796-0.913)$ & $<0.001$ & $0.803(0.741-0.870)$ & $<0.001$ & $0.829(0.764-0.899)$ & $<0.001$ \\
\hline Separated & $0.859(0.710-1.039)$ & 0.118 & $0.821(0.678-0.993)$ & 0.043 & $0.879(0.703-1.100)$ & 0.261 & $0.817(0.652-1.023)$ & 0.077 \\
\hline Single & $0.993(0.915-1.077)$ & 0.864 & $0.966(0.890-1.048)$ & 0.404 & $1.030(0.935-1.134)$ & 0.551 & $0.968(0.879-1.066)$ & 0.512 \\
\hline Widowed & $1.212(1.124-1.308)$ & $<0.001$ & $1.090(1.009-1.178)$ & 0.029 & $1.048(0.955-1.149)$ & 0.322 & $1.073(0.976-1.179)$ & 0.146 \\
\hline Other & $0.987(0.882-1.106)$ & 0.825 & $0.900(0.803-1.009)$ & 0.071 & $0.905(0.788-1.040)$ & 0.159 & $0.861(0.749-0.990)$ & 0.036 \\
\hline T Stage (AJCC, 2004) & & $<0.001$ & & & & $<0.001$ & & \\
\hline TO & Reference & & Reference & & Reference & & Reference & \\
\hline $\mathrm{T} 1$ & $0.273(0.130-0.573)$ & 0.001 & $0.534(0.254-1.124)$ & 0.099 & $0.196(0.088-0.438)$ & $<0.001$ & $0.451(0.201-1.008)$ & 0.052 \\
\hline T2a & $0.193(0.092-0.406)$ & $<0.001$ & $0.498(0.236-1.050)$ & 0.067 & $0.137(0.061-0.307)$ & $<0.001$ & $0.415(0.185-0.931)$ & 0.033 \\
\hline $\mathrm{T} 2 \mathrm{~b}$ & $0.263(0.125-0.553)$ & $<0.001$ & $0.608(0.289-1.280)$ & 0.19 & $0.215(0.096-0.479)$ & $<0.001$ & $0.538(0.240-1.204)$ & 0.131 \\
\hline T3 & $0.313(0.149-0.657)$ & 0.002 & $0.710(0.337-1.496)$ & 0.368 & $0.274(0.123-0.612)$ & 0.002 & $0.664(0.297-1.487)$ & 0.319 \\
\hline T4 & $0.595(0.283-1.250)$ & 0.171 & $0.767(0.364-1.614)$ & 0.484 & $0.538(0.241-1.200)$ & 0.13 & $0.705(0.315-1.577)$ & 0.395 \\
\hline N Stage (AJCC, 2004) & & $<0.001$ & & & & $<0.001$ & & \\
\hline NO & Reference & & Reference & & Reference & & Reference & \\
\hline N1 & 1.269(1.220-1.319) & $<0.001$ & $1.200(1.149-1.253)$ & $<0.001$ & $1.506(1.436-1.580)$ & $<0.001$ & $1.285(1.219-1.355)$ & $<0.001$ \\
\hline N2 & $1.375(1.293-1.463)$ & $<0.001$ & $1.694(1.582-1.813)$ & $<0.001$ & $1.719(1.599-1.848)$ & $<0.001$ & 1.882(1.738-2.039) & $<0.001$ \\
\hline N3 & 1.705(1.548-1.877) & $<0.001$ & $1.874(1.694-2.073)$ & $<0.001$ & $2.171(1.945-2.424)$ & $<0.001$ & $2.147(1.912-2.411)$ & $<0.001$ \\
\hline M Stage (AJCC, 2004) & & $<0.001$ & & & & $<0.001$ & & \\
\hline MO & Reference & & Reference & & Reference & & Reference & \\
\hline M1 & $3.007(2.890-3.128)$ & $<0.001$ & $1.658(1.582-1.737)$ & $<0.001$ & $3.717(3.548-3.893)$ & $<0.001$ & $1.900(1.797-2.008)$ & $<0.001$ \\
\hline Primary Site & & $<0.001$ & & & & $<0.001$ & & \\
\hline Cardia & Reference & & Reference & & Reference & & Reference & \\
\hline Fundus of Stomach & 1.279(1.159-1.413) & $<0.001$ & $1.117(1.011-1.234)$ & 0.03 & $1.201(1.063-1.357)$ & 0.003 & $1.049(0.927-1.186)$ & 0.449 \\
\hline Body of Stomach & 1.093(1.017-1.176) & 0.016 & $1.044(0.968-1.125)$ & 0.264 & $1.022(0.934-1.118)$ & 0.641 & 0.998(0.910-1.095) & 0.966 \\
\hline Gastric Antrum & 0.975(0.926-1.026) & 0.329 & $1.013(0.959-1.071)$ & 0.637 & $0.916(0.861-0.976)$ & 0.006 & 0.993(0.928-1.064) & 0.851 \\
\hline Pylorus & $0.987(0.889-1.096)$ & 0.808 & $1.102(0.990-1.226)$ & 0.075 & $1.006(0.889-1.139)$ & 0.921 & $1.167(1.027-1.326)$ & 0.017 \\
\hline Lesser Curvature of Stomach & $0.833(0.776-0.895)$ & $<0.001$ & $0.869(0.807-0.937)$ & $<0.001$ & $0.765(0.699-0.836)$ & $<0.001$ & $0.823(0.750-0.904)$ & $<0.001$ \\
\hline Greater Curvature of Stomach & $1.087(0.986-1.190)$ & 0.095 & $1.186(1.073-1.310)$ & 0.001 & $1.020(0.904-1.151)$ & 0.747 & $1.144(1.011-1.294)$ & 0.033 \\
\hline Overlapping Lesion of Stomach & 1.478(1.369-1.596) & $<0.001$ & $1.166(1.077-1.263)$ & $<0.001$ & $1.570(1.435-1.717)$ & $<0.001$ & 1.199(1.093-1.315) & $<0.001$ \\
\hline Stomach & $1.339(1.257-1.425)$ & $<0.001$ & $1.110(1.040-1.186)$ & 0.002 & $1.249(1.156-1.349)$ & $<0.001$ & $1.047(0.965-1.135)$ & 0.268 \\
\hline Tumor Size & & $<0.001$ & & & & $<0.001$ & & \\
\hline$\leq 1 \mathrm{~cm}$ & Reference & & Reference & & Reference & & & \\
\hline$>1 \mathrm{~cm}$ & 1.876(1.809-1.946) & $<0.001$ & $1.151(1.105-1.200)$ & $<0.001$ & $1.970(1.885-2.060)$ & $<0.001$ & 1.170(1.113-1.230) & $<0.001$ \\
\hline Gastrectomy & & $<0.001$ & & & & $<0.001$ & & \\
\hline
\end{tabular}




\begin{tabular}{|c|c|c|c|c|c|c|c|c|}
\hline Yes & Reference & & Reference & & Reference & & Reference & \\
\hline No & $3.294(3.172-3.420)$ & $<0.001$ & $2.780(2.648-2.918)$ & $<0.001$ & $3.514(3.358-3.678)$ & $<0.001$ & $2.834(2.671-3.008)$ & $<0.001$ \\
\hline Radiation & & $<0.001$ & & & & $<0.001$ & & \\
\hline Yes & Reference & & & & Reference & & Reference & \\
\hline No & $1.356(1.304-1.410)$ & $<0.001$ & $1.405(1.347-1.466)$ & $<0.001$ & $1.250(1.193-1.309)$ & $<0.001$ & $1.326(1.260-1.395)$ & $<0.001$ \\
\hline
\end{tabular}
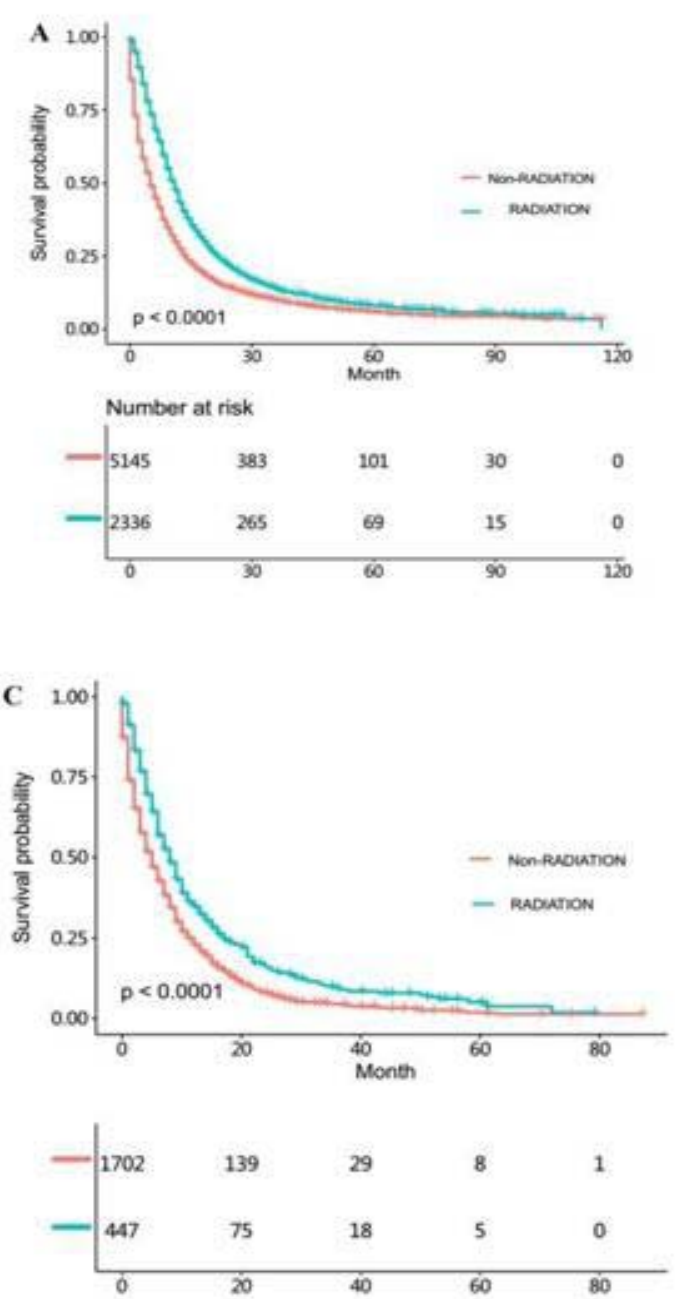
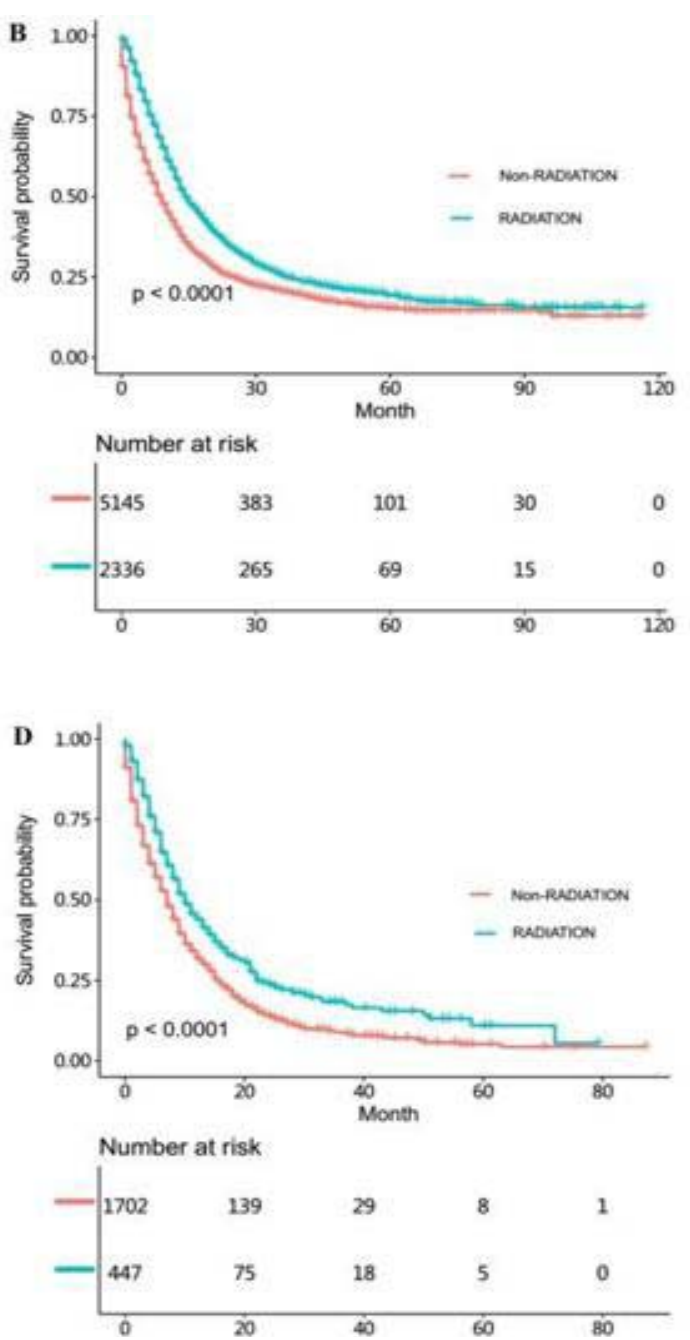

Figure 1: (A) OS time in non-radiotherapy and radiotherapy NSRCC patients. (B) CSS time in non-radiotherapy and radiotherapy NSRCC patients. (C) OS time in non-radiotherapy and radiotherapy SRCC patients. (D) CSS time in non-radiotherapy and radiotherapy SRCC patients.

The influence of radiotherapy on survival time of SRCC and NSRCC patients

In order to further investigate the differences between the effects of radiotherapy on the survival time of SRCC and NSRCC patients, we divided the SRCC and NSRCC patients who were not undergoing gastrectomy into non-radiotherapy NSRCC group, radiotherapy NSRCC group, non-radiotherapy SRCC groupand radiotherapy SRCC group (Table 1 and 2). In Figure 1A-1D, we mainly compared the effects of radiotherapy in non-operative SRCC and NSRCC patients groups. The differences of $\mathrm{OS}(\mathrm{HR}=0.663,95 \% \mathrm{CI}=0.635$ $0.691, \mathrm{p}<0.001)$ and CSS $(\mathrm{HR}=0.668,95 \% \mathrm{CI}=0.636-0.702, \mathrm{p}<0.001)$ time between non-radiotherapy and radiotherapy NSRCC groupwere compared. The results showed that the prognosis of radiotherapy NSRCC groupwas significantly better than non-radiotherapy NSRCC group (Figure 1A and 1B). In addition, we compared the difference of OS $(\mathrm{HR}=0.691,95 \% \mathrm{CI}=0.629-0.758, \mathrm{p}<0.001)$ and CSS $(\mathrm{HR}=0.710$, 95\% CI $=0.638-0.789, \mathrm{p}<0.001)$ time between non-radiotherapy and radiotherapy SRCC group, which showed that the prognosis of radiotherapy SRCC groupwas better than non-radiotherapy SRCC group. Based on the above results, we can learn that radiotherapy benefits the prognosis of both SRCC and NSRCC patients. In order to eliminate the effects of other factors, we re-compared the OS and CSS time of the non-radiotherapy and radiotherapy NSRCC groupthrough PSM. In Figure 2A, 2B and 3, we compared the OS and 

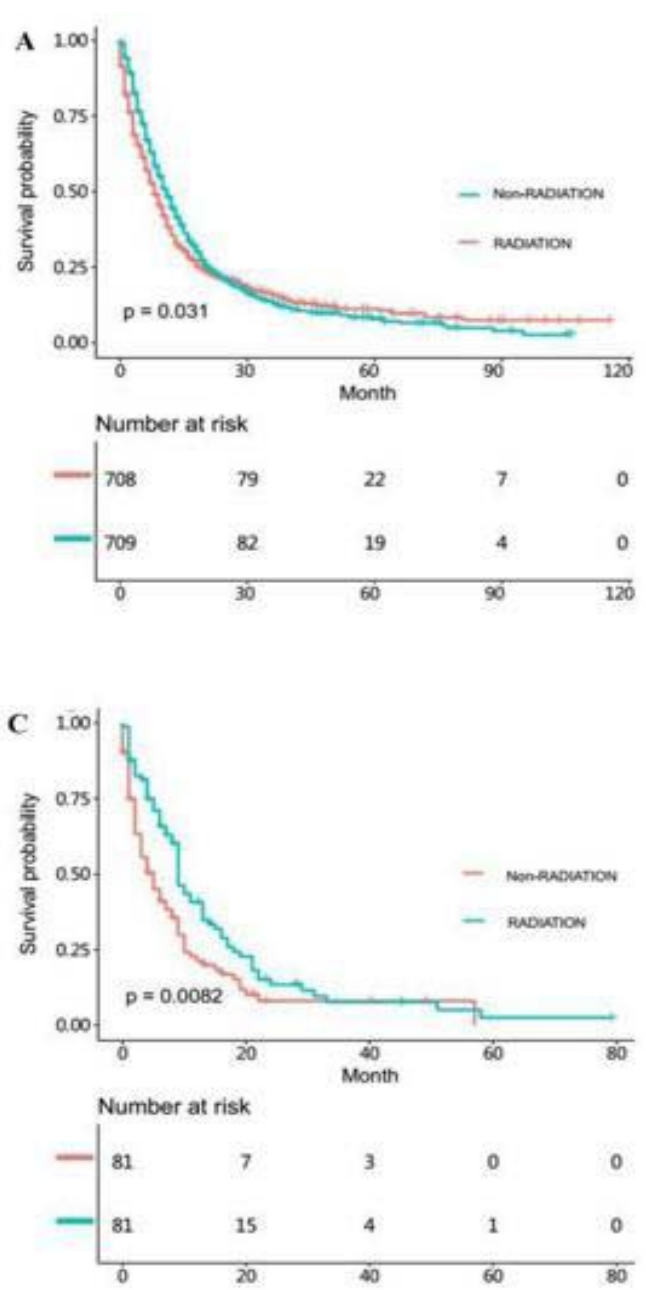
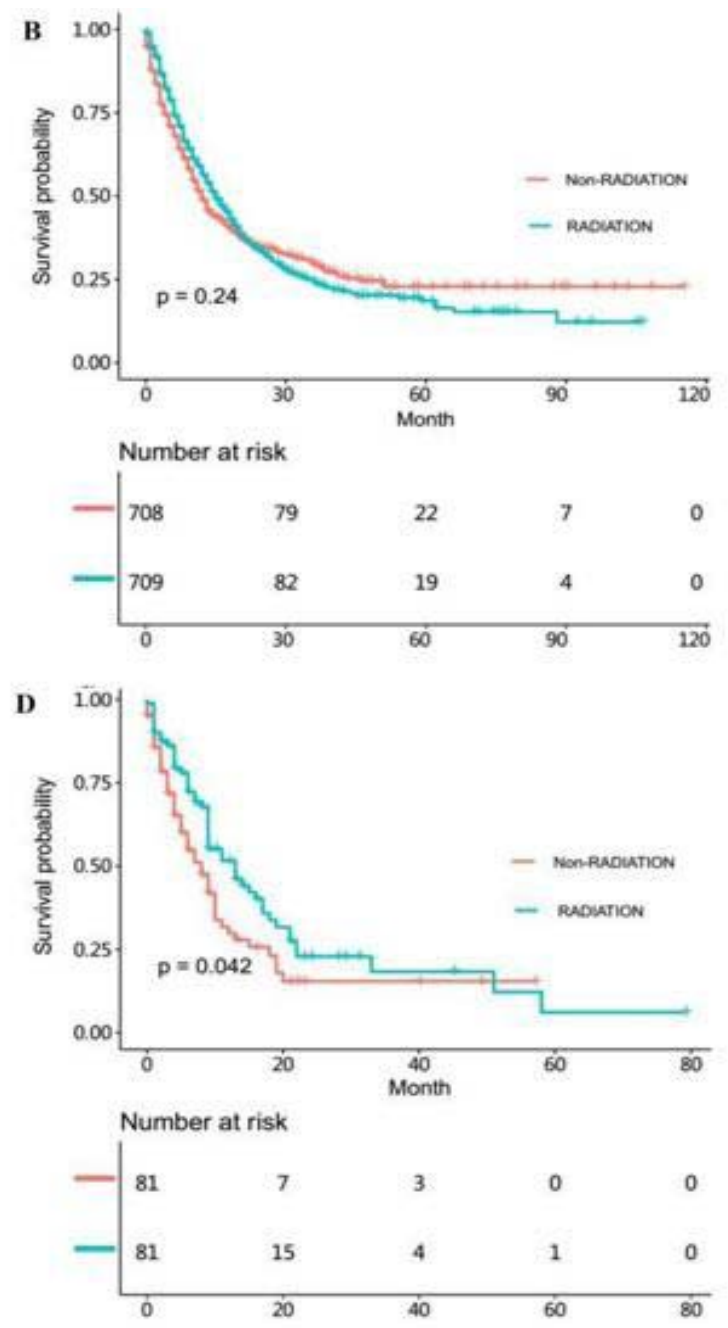

Figure 2: (A) OS time in non-radiotherapy and radiotherapy NSRCC patients after PSM analysis. (B) CSS time in non-radiotherapy and radiotherapy NSRCC patients after PSM analysis. (C) OS time in non-radiotherapy and radiotherapy SRCC patients after PSM analysis. (D) CSS time in non-radiotherapy and radiotherapy SRCC patients after PSM analysis.

CSS time after PSM in non-radiotherapy and radiotherapy NSRCC group. According to results reflected in Figure 2A, the OS time in radiotherapy NSRCC groupwas better than in non-radiotherapy NSRCC group $(\mathrm{HR}=0.882,95 \% \mathrm{CI}=0.785-0.992, \mathrm{P}=0.037)$, but the lifetime curves was intersected. We then segmented the statistical data and the results showed that the OS time in two groups had no statistical difference (Figure 3). And there was no statistical difference in the CSS time between radiotherapy and non-radiotherapy NSRCC group $(\mathrm{HR}=0.921,95 \% \mathrm{CI}=0.800-1.060, \mathrm{p}=0.252) \quad$ (Figure 2B). Next, we also compared the OS and CSS time after PSM in nonradiotherapy and radiotherapy SRCC group, and the $\mathrm{OS}(\mathrm{HR}=0.649$, 95\% $\mathrm{CI}=0.462-0.910, \mathrm{p}=0.012)$ and CSS $(\mathrm{HR}=0.674,95 \% \mathrm{CI}=0.453$ $0.901, \mathrm{p}=0.042$ ) time in radiotherapy SRCC groupwere better than that in non-radiotherapy SRCC group. From the above data, we can initially get the conclusion that radiotherapy for SRCC patients may be more effective than NSRCC patients, with statistical differences.

The influence of radiotherapy and gastrectomy on survival time in SRCC and NSRCC patients

For patients undergoing gastrectomy, they were also divided into non-radiotherapy NSRCC group, radiotherapy NSRCC group, nonradiotherapy SRCC groupand radiotherapy SRCC group (Table 3 and 4). In Figure 4A-4D and Figure 5 and 6, we mainly compared the effects of radiotherapy on the SRCC and NSRCC patients with gastrectomy. As reflected in Figure 4A, the OS time in radiotherapy NSRCC groupis better than that in non-radiotherapy NSRCC group $(\mathrm{HR}=0.771,95 \% \mathrm{CI}=0.729-0.816, \mathrm{p}<0.0001)$. And the CSS time in radiotherapy NSRCC groupwas better than that in non-radiotherapy NSRCC group $(\mathrm{HR}=0.915,95 \% \mathrm{CI}=0.855-0.979, \mathrm{p}=0.01)$, but the lifetime curves was also intersected (Figure 4B). We then segmented the statistical analysis data and the results showed that the CSS time in radiotherapy NSRCC groupwas better than that in non-radiotherapy NSRCC groupduring the first two years, but after the period time the CSS time in non-radiotherapy NSRCC groupwas better than that in radiotherapy NSRCC group.

We next compared the OS time in non-radiotherapy and radiotherapy SRCC groups, the results demonstrated that OS time in radiotherapy SRCC groupwas better than that in non-radiotherapy SRCC group $(H R=0.792,95 \% \mathrm{CI}=0.730-0.860, \mathrm{p}<0.0001)$ (Figure $4 \mathrm{C})$. 


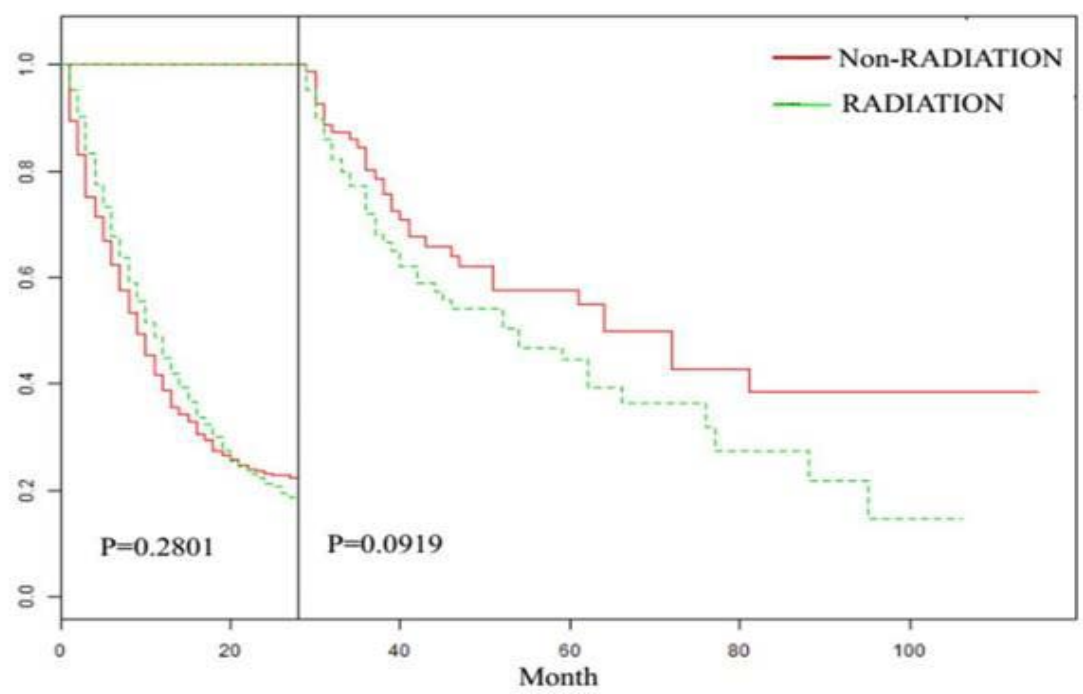

Figure 3: Landmark Estimation of OS time in non-radiotherapy and radiotherapy NSRCC patients after PSM analysis.
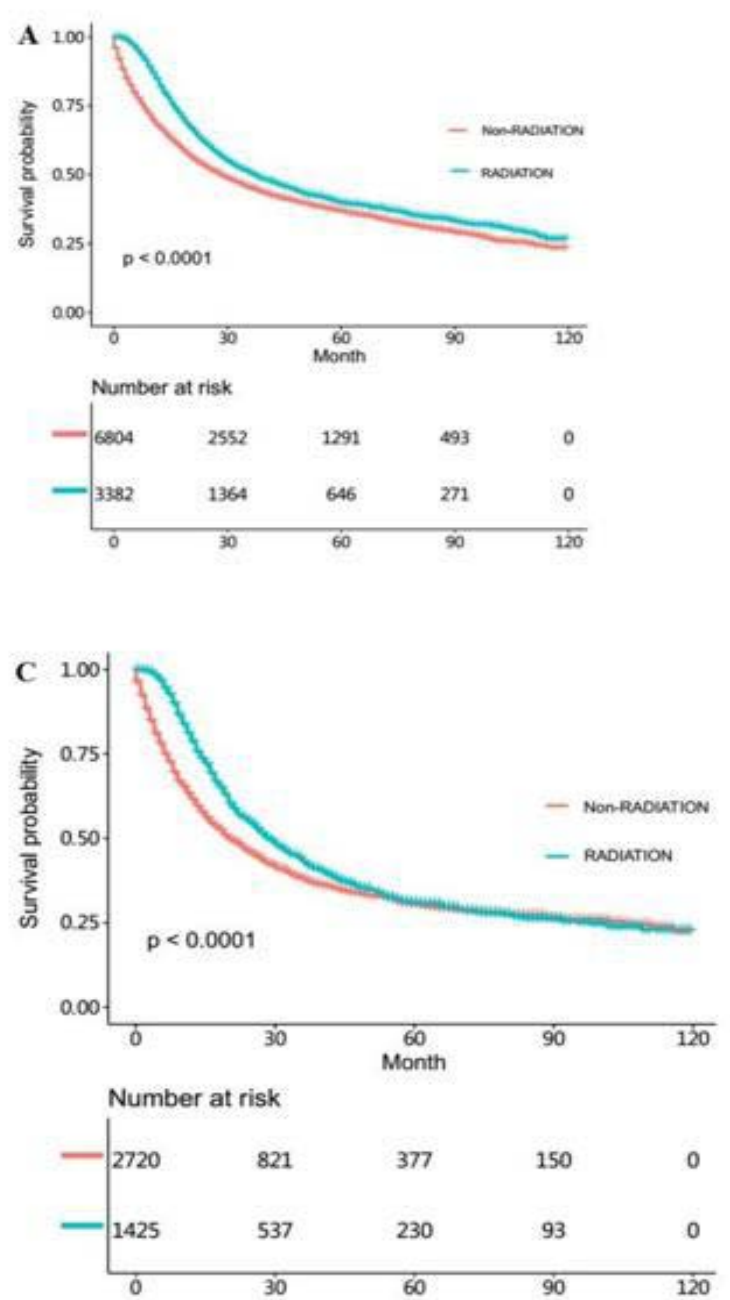
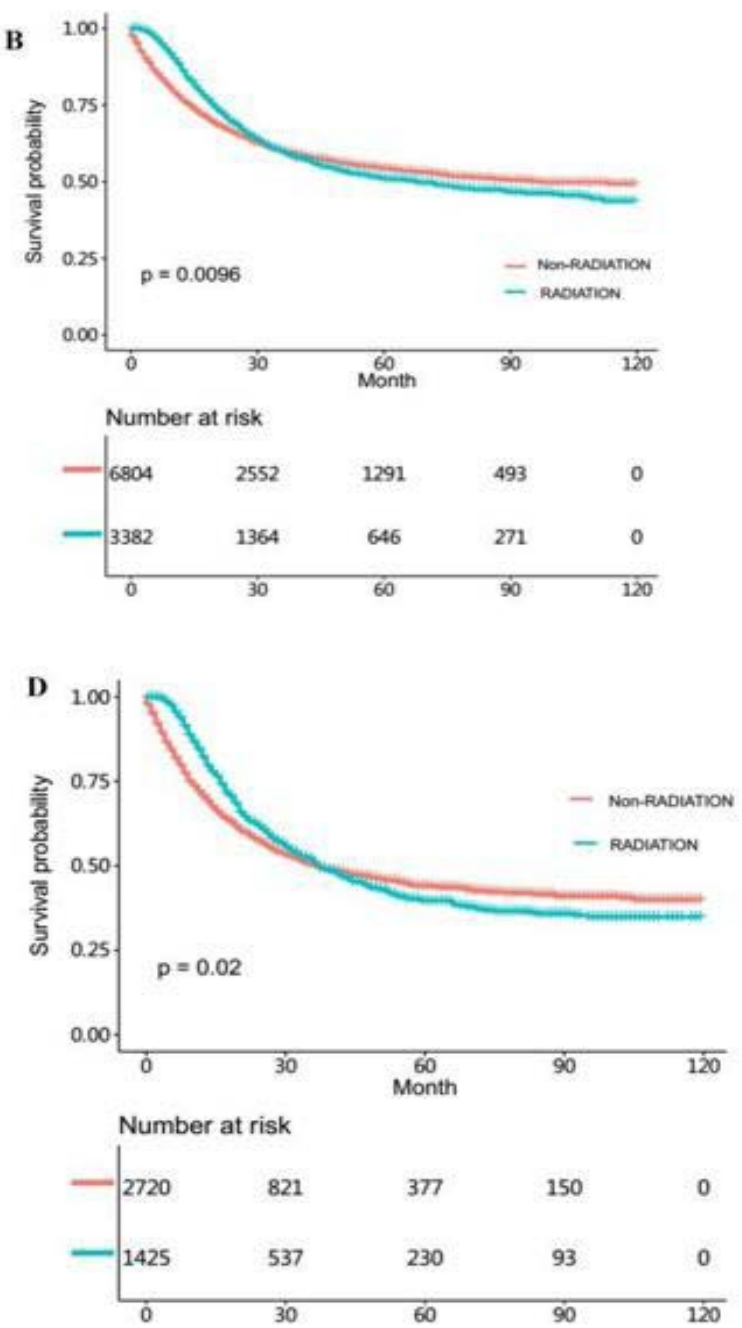

Figure 4: (A) OS time in non-radiotherapy and radiotherapy NSRCC patients under gastrectomy treatment. (B) CSS time in non-radiotherapy and radiotherapy NSRCC patients undergoing gastrectomy treatment. (C) OS time in non-radiotherapy and radiotherapy SRCC patients under gastrectomy treatment. (D) CSS time in non-radiotherapy and radiotherapy SRCC patients under gastrectomy treatment. 


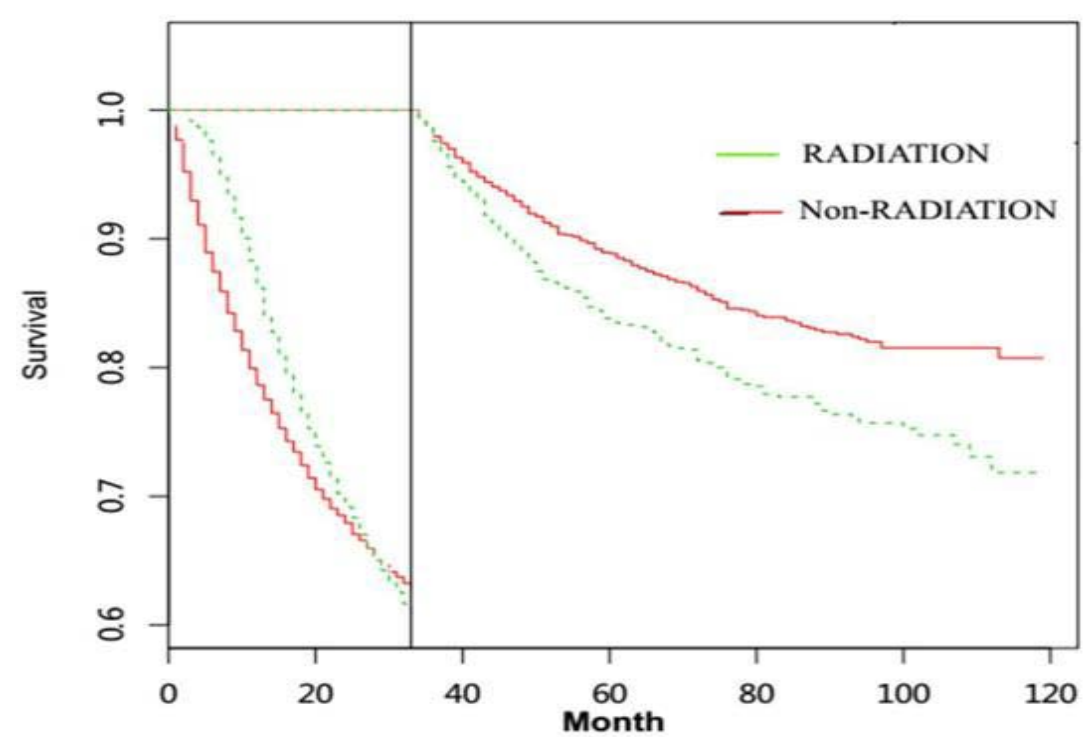

Figure 5: Landmark Estimation of CSS time in non-radiotherapy and radiotherapy NSRCC patients under gastrectomy treatment

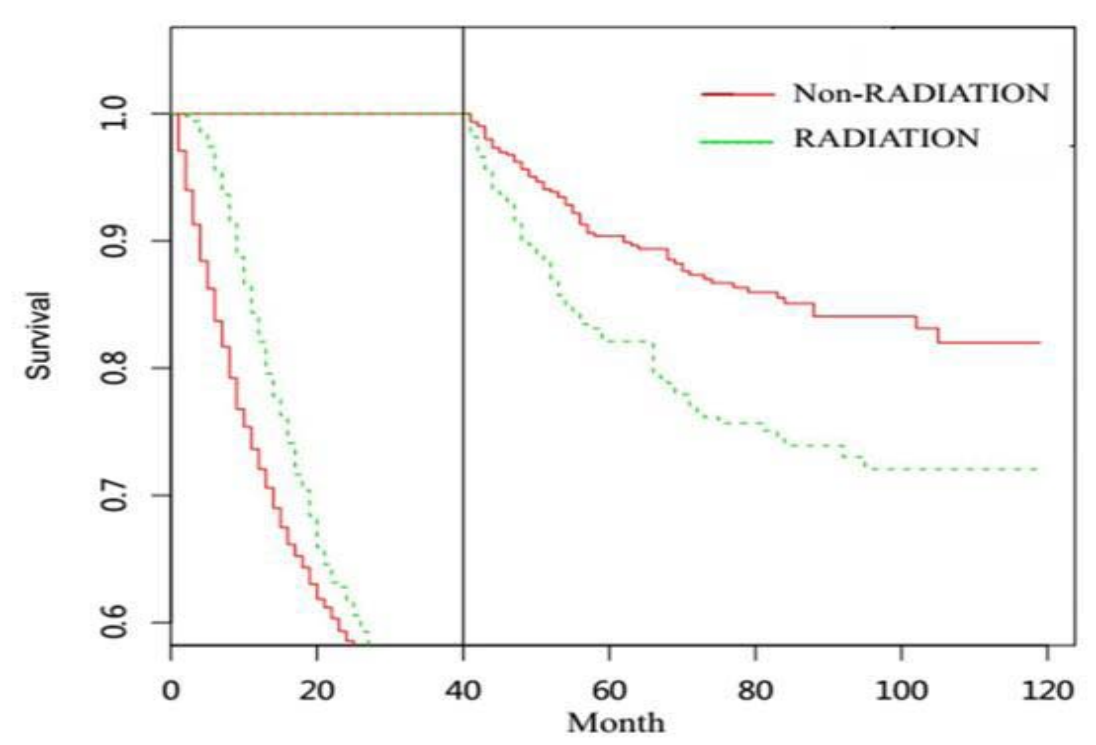

Figure 6: Landmark Estimation of CSS time in non-radiotherapy and radiotherapy SRCC patients under gastrectomy treatment.

As shown in Figure 4D, the CSS time in radiotherapy SRCC groupwas also better than in non-radiotherapy SRCC group $(\mathrm{HR}=0.895,95 \%$ $\mathrm{CI}=0.814-0.984, \mathrm{p}=0.021$ ), but the lifetime curves was intersected. We then segmented the statistical analysis data and the result indicated that during the first 40 months the CSS time in radiotherapy SRCC groupwas better than that in non-radiotherapy SRCC group, but after that period time, the CSS in non-radiotherapy SRCC groupwas better than that in radiotherapy SRCC groupwhich had statistical difference (Figure 6). By comparing the survival time of these groups, we could conclude that radiotherapy did not benefit all patients, especially for those undergoing gastrectomy.

In order to eliminate the imbalanced factors of the two groups we further analyzed the data by PSM method. The OS and CSS time of the matched non-radiotherapy and radiotherapy NSRCC groupwere then compared. From the results, we could learn that there was no significant statistical differences in OS ( $\mathrm{HR}=0.939,95 \% \mathrm{CI}=0.698$ 1.263, $\mathrm{p}=0.730$ ) and CSS (HR=0.866, 95\% CI $=0.604-1.240, \mathrm{p}=0.410)$ time between the non-radiotherapy and radiotherapy NSRCC groups (Figure 7A and 7B). And we also compared the OS and CSS time in the non-radiotherapy and radiotherapy SRCC groupand the results demonstrated that the $\mathrm{OS}(\mathrm{HR}=0.719,95 \% \mathrm{CI}=0.584-0.883$, $\mathrm{p}=0.0014)$ and CSS (HR=0.718, 95\% CI $=0.566-0.912, \mathrm{p}=0.0059)$ time in the radiotherapy SRCC groupwere both better than those in the non-radiotherapy SRCC group.

\section{Discussion}

Based on the analysis data obtained from SEER database, we discuss the clinical value of radiotherapy in SRCC and NSRCC patients in this study. Through the multivariate analysis and 

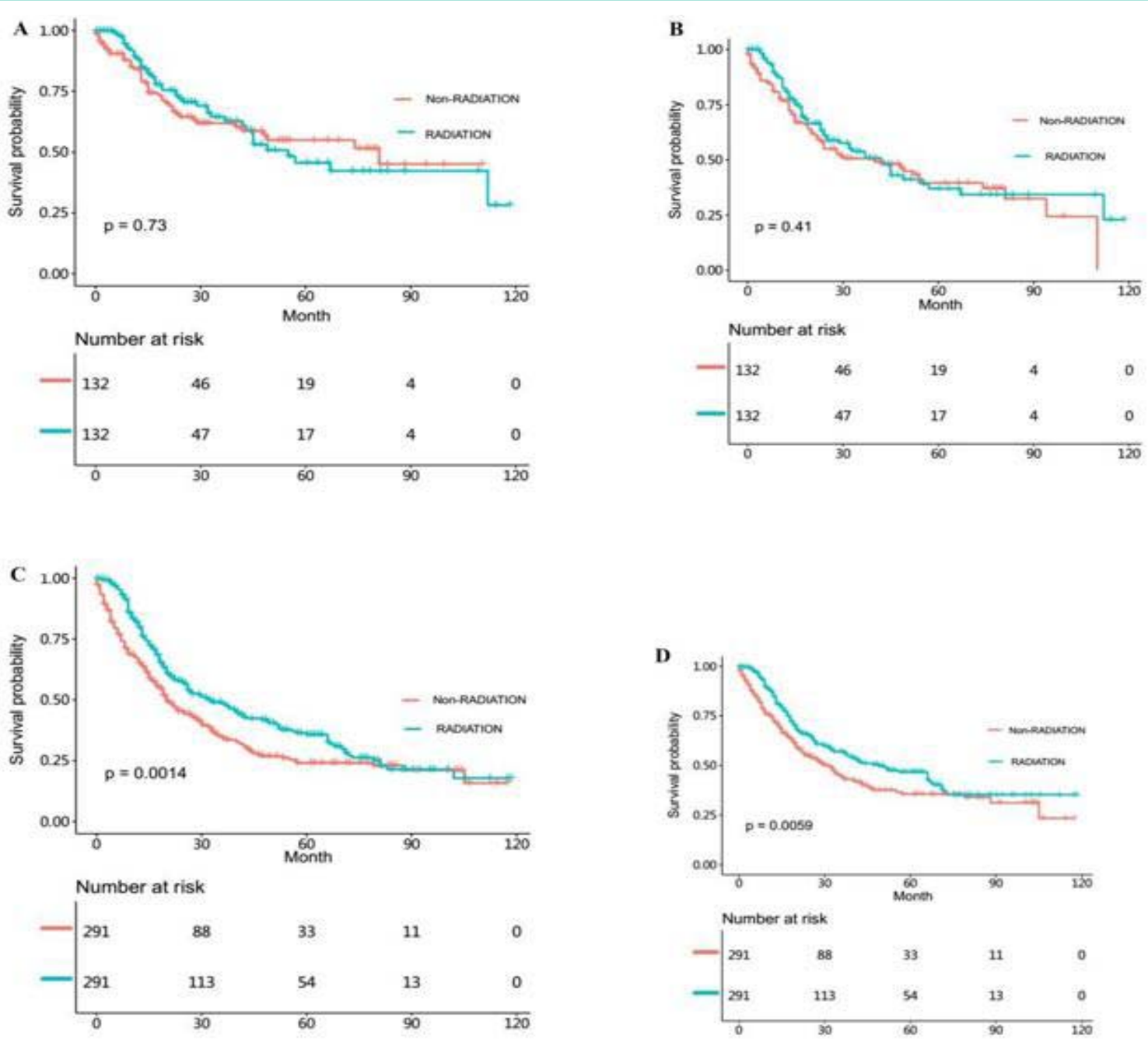

Figure 7: (A) OS time in non-radiotherapy and radiotherapy NSRCC patients under gastrectomy treatment after PSM analysis. (B) CSS time in non-radiotherapy and radiotherapy NSRCC patients undergoing gastrectomy treatment after PSM analysis. (C) OS time in non-radiotherapy and radiotherapy SRCC patients under gastrectomy treatment after PSM analysis. (D) CSS time in non-radiotherapy and radiotherapy SRCC patients under gastrectomy treatment after PSM analysis.

univariate analysis strategies, we found that radiotherapy is of obvious value to both SRCC and NSRCC patients. However, the effect of radiotherapy on the prognosis of SRCC patients was better than that in NSRCC patients by using univariate analysis. In order to study the reasons for the differences, we divided the patient into different groups based on whether they received gastrectomy. According to the OS and CSS time, we could learn that radiotherapy had benefits on non-gastrectomy SRCC groupand NSRCC grouppatients. In order to eliminate the multivariate factors, we then compared the groups with PSM matching method. The radiotherapy had no effect on the OS and CSS in NSRCC group, while radiotherapy had obvious benefit for SRCC group. Next, we studied the effects of gastrectomy combined with radiotherapy on SRCC groupand NSRCC grouppatients and we have found that the OS time was significantly improved in SRCC groupand NSRCC groupunder gastrectomy and radiotherapy treatment. However, the effect in SRCC groupand NSRCC groupwas reversed after a period time of 40 months and 32 months, respectively. So we further compared them with PSM. Data showed that radiotherapy had no effect on the OS and CSS time in NSRCC group, while radiotherapy had obvious benefits for SRCC group. In this study, we got the conclusion that SRCC patients was more sensitive radiotherapy than NSRCC patients were, which had never been reported before.

For early-stage gastric cancer patients, gastrectomy is the main treatment to achieve the goal of $\mathrm{R} 0$ resection and the surgical margin less than $4 \mathrm{~cm}$. But the role of radiotherapy in the treatment of gastric cancer patients is still controversial. Then two randomized trials compared the effects of surgery together with radiotherapy or chemotherapy were conducted in the premise that gastric cancer patients received resection. A gastric cancer study groupin the UK randomly divided patients into gastrectomy or gastrectomy combined with radiotherapy groups, although there was a significant reduction in the recurrence of local lesions in patients undergoing radiotherapy, no survival benefits were found [16]. Zhang [17] divided the patients into preoperative radiotherapy treatment and surgery alone groups and reported that radiotherapy could improve survival rates and surgical resection rates. A recent meta-analysis assessed the role of preoperative, postoperative and intraoperative 
radiotherapy in resectable gastric carcinoma patients and results showed that patients undergoing radiotherapy had significantly improved OS time in 5 years [18]. Through Cox regression analysis in our study, it could be found that radiotherapy has benefited both the SRCC and NSRCC grouppatients, but according to the classification treatment and survival time comparison, we found that radiotherapy benefits for NSRCC patients seem inferior to SRCC patients. The benefits of radiotherapy for the SRCC groupare certain and no similar studies have been carried out in the relevant literature. However, the molecular biological differences between the two groups remained unclear.

SRCC is a more common type of gastric adenocarcinoma with more than $50 \%$ of tumor cells are rich in intracellular mucus. Through this study, it can be found that there were many obvious differences in clinic pathological features of SRCC and NSRCC patients. For example, SRCC patients are mostly women, have younger age, lower tumor differentiation and higher incidence of lymphatic and distant metastasis [19]. These characteristics of SRCC patients might be the reasons of making their prognosis significantly lower than NSRCC patients. Compared to the formation process of NSRCC, SRCC mainly has two pathological processes of cell adhesion molecules loss and accumulations of mucus into large vacuoles in the cellular level. E-cadherin protein encoded by CDH1 gene is a cell-cell adhesion molecule, which plays a key role in tumor progression and epithelial mesenchymal transformation. And the increased E-cadherin protein expression is an important reason for the occurrence of SRCC [20]. Moreover, E-cadherin is a key component of epithelial cell adhesion connections and is necessary for the normal formation and maintenance of epithelial cells [21]. E-cadherin protein reduction is an important factor leading to the invasion and metastasis of epithelial tumors [22]. During the course of tumor progression, various mechanisms might lead to decreased E-cadherin level, which leads to the destruction of normal cell adhesion and enhances the movement and invasion of tumor cells [23]. E-cadherin expression is also closely related to the degree of differentiation in gastric cancers. Almost all the relevant studies showed that the expression level of E-cadherin in gastric cancer tissue with well differentiation was significantly higher than that with poor differentiation or no differentiation. Karayiannakis's [24] study of 83 gastric cancer cases showed that the abnormal expression of E-cadherin in adenocarcinoma was $57 \%$, while the abnormal expression level in ring cell carcinoma and undifferentiated carcinoma were $84 \%$ and $87 \%$, respectively. This may be an important reason for the poor differentiation and distant metastasis of SRCC. Ray's [25] study on radiotherapy sensitivity through comparing E-cadherin expression in prostate cancer patients found that the decline in E-cadherin expression was associated with a decreased sensitivity to radiotherapy, suggesting that E-cadherin expression could promote the treatment effect of radiotherapy in prostate cancer patients, which mainly illustrates the role of E-cadherin expression in radiotherapy sensitivity, but the specific mechanism has not been further studied.

Our study incorporates a large number of patients from multi-center population data in United States, avoiding related bias caused by a single agency experience or a limited sample size. But due to the non-stochastic nature of SEER, some limitations of current research are worth discussing. First, it is not feasible to review individual pathological diagnoses in large populations and interpretation differences between pathologists may lead to incorrect classification. Second, the SEER registration form does not include detailed information on the dose of radiotherapy and chemotherapy, including PRT or the durations of radiotherapy and chemotherapy. Therefore, we are unable to take the differences in radiotherapy treatment into account during the study period. Although there is no data on cancer recurrence in this study, CSS is a reasonable index to evaluate the prognosis of gastric cancer patients. The results of the current study may provide some scientific information for future research on the specific effect of radiotherapy on SRCC patients. In order to get a more explicit conclusion, we will conduct a larger randomized and controlled trial of the Chinese population through multi-center cooperation method.

To sum up, our results show that compared to NSRCC, SRCC is a unique type of gastric cancer, which is more likely to affect younger patients, have features such as manifested in more advanced tumors and less differentiated in diagnosis. The sensitivity of SRCC to radiotherapy is higher than NSRCC patients. Although the prognosis of SRCC patients is far less than that of NSRCC patients, the treatment strategy of surgical resection of primary lesions combined with radiotherapy should be recommend for SRCC patients. And whether radiotherapy benefits NSRCC patients still needs further to be explored.

\section{Availability of Data and Materials}

In this study, the clinical value of radiotherapy for SRCC and NSRCC patients using SEER database (http://seer.cancer.gov/about/ overview.html), which is maintained by the National Cancer Institute and includes 18 population-based cancer registries with information of cancer morbidity and mortality in the United States.

Lei $\mathrm{Yu}$ conceived and designed the study; Chunming Wang, Huafu Li and Jianwu Luo performed the data statistics; Huafu Li and Riqiang Liu wrote the paper. All authors read and approved the final manuscript.

\section{References}

1. Bray F, Ferlay J, Soerjomataram I, Siegel RL, Torre LA, Jemal A. Global cancer statistics 2018: GLOBOCAN estimates of incidence and mortality worldwide for 36 cancers in 185 countries. CA: a cancer journal for clinicians. 2018; 68: 394-424.

2. Lauren P. The Two Histological Main Types of Gastric Carcinoma: Diffuse and So-Called Intestinal-Type Carcinoma. An Attempt at a Histo-Clinical Classification. Acta pathologica et microbiologica Scandinavica. 1965; 64: 31-49.

3. Japanese classification of gastric carcinoma: $3^{\text {rd }}$ English edition. Gastric cancer: official journal of the International Gastric Cancer Association and the Japanese Gastric Cancer Association. 2011; 14: 101-112.

4. Ming SC. Gastric carcinoma. A pathobiological classification. Cancer. 1977 39: 2475-2485.

5. Fukui Y. Mechanisms behind signet ring cell carcinoma formation. Biochemica and biophysical research communications. 2014; 50: 1231-1233.

6. Bamboat ZM, Tang LH, Vinuela E, et al. Stage-stratified prognosis of signet ring cell histology in patients undergoing curative resection for gastric adenocarcinoma. Annals of surgical oncology. 2014; 21: 1678-1685.

7. Liu X, Cai $H$, Sheng $W$, et al. Clinicopathological Characteristics and Survival Outcomes of Primary Signet Ring Cell Carcinoma in the Stomach: Retrospective Analysis of Single Center Database. PloS one. 2015; 10: 
e0144420.

8. Gronnier C, Messager M, Robb WB, et al. Is the negative prognostic impact of signet ring cell histology maintained in early gastric adenocarcinoma? Surgery. 2013; 154: 1093-1099.

9. Ajani JA, D'Amico TA, Almhanna K, et al. Gastric Cancer, Version 3.2016, NCCN Clinical Practice Guidelines in Oncology. Journal of the National Comprehensive Cancer Network: JNCCN. 2016; 14: 1286-1312.

10. Min C, Bangalore S, Jhawar S, et al. Chemoradiation therapy versus chemotherapy alone for gastric cancer after RO surgical resection: a metaanalysis of randomized trials. Oncology. 2014; 86: 79-85.

11. Noel G, Jauffret E, Mazeron JJ. [Randomized clinical trial on the combination of preoperative irradiation and surgery in the treatment of adenocarcinoma of gastric cardia (AGC)--report on 370 patients]. Cancer radiotherapie: journal de la Societe francaise de radiotherapie oncologique. 1999; 3: 344.

12. Wu SG, Zhang WW, Sun JY, He ZY, Su GQ, Li FY. Preoperative radiotherapy improves survival in rectal signet-ring cell carcinoma-a population-based study. 2017; 12: 141

13. Ling CR, Wang R, Wang MJ, Ping J, Zhuang W. Prognosis and value of preoperative radiotherapy in locally advanced rectal signet-ring cell carcinoma. Scientific reports. 2017; 7: 45334.

14. Sun M, Trinh QD. A Surveillance, Epidemiology and End Results (SEER) database malfunction: perceptions, pitfalls and verities. BJU international. 2016; 117: 551-552.

15. Parast L, Tian L, Cai T. Landmark Estimation of Survival and Treatment Effect in a Randomized Clinical Trial. Journal of the American Statistical Association. 2014; 109: 384-394.

16. Austin PC. Optimal caliper widths for propensity-score matching when estimating differences in means and differences in proportions in observational studies. Pharmaceutical statistics. 2011; 10: 150-161.

17. Hallissey MT, Dunn JA, Ward LC, Allum WH. The second British Stomach Cancer Group trial of adjuvant radiotherapy or chemotherapy in resectable gastric cancer: five-year follow-up. Lancet (London, England). 1994; 343: 1309-1312.

18. Zhang M, Zhu G, Zhang H, Gao H, Xue Y. Clinicopathologic features of gastric carcinoma with signet ring cell histology. Journal of gastrointestinal surgery: official journal of the Society for Surgery of the Alimentary Tract. 2010; 14: 601-606.

19. Valentini V, Cellini F, Minsky BD, et al. Survival after radiotherapy in gastric cancer: systematic review and meta-analysis. Radiotherapy and oncology: journal of the European Society for Therapeutic Radiology and Oncology. 2009; 92: 176-183.

20. Bozkaya Y, Erdem GU, Ozdemir NY, et al. Comparison of clinicopathological and prognostic characteristics in patients with mucinous carcinoma and signet ring cell carcinoma of the stomach. Current medical research and opinion. 2017; 33: 109-116.

21. Humar B, Blair V, Charlton A, More H, Martin I, Guilford P. E-cadherin deficiency initiates gastric signet-ring cell carcinoma in mice and man. Cancer research. 2009; 69: 2050-2056.

22. Gul IS, Hulpiau P, Saeys Y, van Roy F. Evolution and diversity of cadherins and catenins.Experimental cell research. 2017; 358: 3-9.

23. Zhao $\mathrm{H}$, Zhang $\mathrm{D}$, Yang L, Wang E. p0071 interacts with E-cadherin in the cytoplasm so as to promote the invasion and metastasis of non-small cell lung cancer. Molecular carcinogenesis. 2018; 57: 89-96.

24. Canel M, Serrels A, Frame MC, Brunton VG. E-cadherin-integrin crosstalk in cancer invasion and metastasis. Journal of cell science. 2013; 126: 393-401.

25. Karayiannakis AJ, Syrigos KN, Chatzigianni E, Papanikolaou S, Karatzas GJH. E-cadherin expression as a differentiation marker in gastric cancer. 1998; 45: 2437-2442.

26. Ray ME, Mehra R, Sandler HM, Daignault S, Shah RB. E-cadherin protein expression predicts prostate cancer salvage radiotherapy outcomes. The Journal of urology. 2006; 176: 1409-1414; Discussion 1414. 